\title{
Nonequilibrium velocity fluctuations and energy amplification in planar Couette flow
}

\author{
José M. Ortiz de Zárate* \\ Departamento de Física Aplicada I, Facultad de Física, Universidad Complutense, 28040 Madrid, Spain \\ Jan V. Sengers \\ Institute for Physical Science and Technology and Burgers Program for Fluid Dynamics, University of Maryland, College Park, \\ Maryland 20742-8510, USA
}

(Received 8 January 2009; published 7 April 2009)

\begin{abstract}
In this paper we investigate intrinsic thermally excited nonequilibrium velocity fluctuations in laminar planar Couette flow. For this purpose we have complemented the solution of the stochastic Orr-Sommerfeld equation for the intensity of the fluctuations of the wall-normal velocity, presented in a previous publication, with a solution of the stochastic Squire equation for the intensity of the fluctuations of the wall-normal vorticity. We have obtained exact solutions of these equations without boundary conditions and solutions in a Galerkin approximation when appropriate boundary conditions are included. These results enable us to make a quantitative assessment of the intensity of these nonequilibrium fluctuations, as well as of the related energy amplification, which are always present, even in the absence of any externally imposed noise.
\end{abstract}

DOI: 10.1103/PhysRevE.79.046308

PACS number(s): 47.15.-x, 05.20.Jj, 05.40.-a, 05.70.Ln

\section{INTRODUCTION}

The purpose of the present paper is to investigate velocity fluctuations in a liquid under steady shear on the basis of fluctuating hydrodynamics. The validity of the method of fluctuating hydrodynamics for dealing with thermally excited fluctuations in stationary nonequilibrium states is now well established, as reviewed in a recent monograph [1]. Fluctuations in nonequilibrium states turn out to be very different from what one would expect on the basis of a localequilibrium assumption for the fluctuations. While thermal fluctuations in fluids in thermodynamic equilibrium states are spatially short ranged except for states near a critical point, thermal fluctuations are always spatially long ranged in nonequilibrium states [2]. Moreover, the intensity of the fluctuations exhibits a significant enhancement in nonequilibrium states, even far away from any hydrodynamic instability. Nonequilibrium enhancements of thermal fluctuations in fluids in the presence of a temperature and/or a concentration gradient have been observed experimentally, both with light scattering [3-7] and with shadowgraphy [8-14].

In the case of a liquid between two horizontal plates and subjected to a stationary temperature gradient the thermal fluctuations not only exhibit a pronounced nonequilibrium enhancement when the liquid layer is heated from below [15-17] but also when the layer is heated from above [2,3], as originally predicted by Kirkpatrick et al. [18]. The intensity of the nonequilibrium enhancement of the (temperature) fluctuations increases with increasing values of the Rayleigh number. Eventually the intensity of the nonequilibrium fluctuations, predicted by fluctuating hydrodynamics, diverges at a value of the Rayleigh number consistent with the critical value of the Rayleigh number that follows from traditional hydrodynamic instability analysis for the onset of convection [19].

\footnotetext{
*jmortizz@ fis.ucm.es
}

For a liquid under steady shear one needs to evaluate the (velocity) fluctuations as a function of the Reynolds number. A fundamental problem is that in the case of planar Couette and planar Poiseulle flow hydrodynamic instability analysis does not yield a critical value of the Reynolds number that would be associated with a transition from laminar to turbulent flow [20-22]. As a consequence, many investigators have studied the effect of external perturbations on the flow. For incompressible flow this is commonly done on the basis of the so-called Orr-Sommerfeld and Squire equations, which are equations for the vertical components of the velocity and vorticity, also referred to as (wall-) normal velocity and (wall-) normal vorticity [20,21]. Specifically, one considers solutions of the (deterministic) Orr-Sommerfeld and Squire equations after imposing various external perturbations [23-35].

Here we approach the problem on the basis of fluctuating hydrodynamics. Hence, we shall not consider the effects of any external perturbations, but shall evaluate the intrinsic velocity fluctuations that always are present, even in the absence of any external noise. For this purpose we have formulated stochastic versions of the Orr-Sommerfeld and Squire equations. In a previous publication we have obtained the solution of the stochastic Orr-Sommerfeld equation for the fluctuations of the wall-normal component of the velocity in the planar Couette flow problem [36]. This solution is a generalization of solutions obtained by some previous investigators $[37,38]$ by incorporating also the effect of the boundary conditions at the planar walls for the velocity fluctuations. As the next step we need to complement our previous results with a solution of the stochastic Squire equation for the fluctuations of the wall-normal component of the vorticity. The nonequilibrium enhancement of the fluctuations of the wallnormal velocity is caused by a coupling of the corresponding hydrodynamic mode with different wave numbers. To determine the nonequilibrium enhancement of the fluctuations of the wall-normal vorticity we need also to account for a coupling of these vorticity fluctuations with the wall-normalvelocity fluctuations with the same wave number. By com- 
bining the solutions of the stochastic Orr-Sommerfeld and Squire equations, we shall be able to determine the nonequilibrium enhancement of the (kinetic) energy which is related to the concept of energy amplification in the fluid-dynamics literature [32].

We shall proceed as follows. In Sec. II we first remind the reader of the theory of fluctuations in an incompressible liquid in the absence of flow. In Sec. III we specify the stochastic Orr-Sommerfeld and Squire equations. To obtain a comprehensive solution for the fluctuations we need to supplement the stochastic Orr-Sommerfeld and Squire equations with appropriate boundary conditions for the velocity and vorticity fluctuations. First in Sec. IV we consider the solutions of the stochastic Orr-Sommerfeld and Squire equations in the absence of such boundary conditions. Then in Sec. V we consider how these solutions are modified by appropriate boundary conditions for the normal-velocity and normal-vorticity fluctuations. In Sec. VI we determine the intrinsic nonequilibrium energy amplification resulting from the velocity fluctuations. We conclude the paper with some final remarks in Sec. VII.

\section{VELOCITY FLUCTUATIONS IN EQUILIBRIUM}

In this section we briefly review the theory for the velocity autocorrelation functions in an incompressible liquid in equilibrium, i.e., in the absence of any macroscopic flow. For clarity we use in this section variables with physical dimensions, while in the remainder of the paper we use mostly dimensionless variables, as we did in our previous publication on the subject [36]. The relevant linearized fluctuatinghydrodynamics equations for the fluctuations $\delta \mathbf{v}(\mathbf{r}, t)$ and $\delta p(\mathbf{r}, t)$ of the velocity and the pressure at location $\mathbf{r}$ and time $t$ around their mean values $\mathbf{v}=\mathbf{v}_{0}=0$ and $p=p_{0}$ are

$$
\begin{gathered}
\boldsymbol{\nabla} \cdot \delta \mathbf{v}=0, \\
\frac{\partial(\delta \mathbf{v})}{\partial t}=-\frac{1}{\rho_{0}} \nabla \delta p+\nu \nabla^{2} \delta \mathbf{v}+\frac{1}{\rho_{0}} \nabla \cdot \delta \Pi,
\end{gathered}
$$

where $\rho_{0}$ is the (mean) density, $\nu$ is the kinematic viscosity, and $\delta \Pi(\mathbf{r}, t)$ is a random fluctuating stress tensor [1,39]. The autocorrelation function for the random stress tensor for a fluid in equilibrium is given by the fluctuation-dissipation theorem. For an incompressible liquid under consideration here, the autocorrelation function between the different components of the random stress tensor is $[1,39,40]$

$$
\begin{aligned}
& \left\langle\delta \Pi_{i j}(\mathbf{r}, t) \cdot \delta \Pi_{k l}\left(\mathbf{r}^{\prime}, t^{\prime}\right)\right\rangle \\
& \quad=2 k_{B} T \eta\left(\delta_{i k} \delta_{j l}+\delta_{i l} \delta_{j k}\right) \delta\left(\mathbf{r}-\mathbf{r}^{\prime}\right) \delta\left(t-t^{\prime}\right),
\end{aligned}
$$

where $k_{B}$ is Boltzmann's constant, $T$ is the temperature, $\eta$ $=\rho_{0} \nu$ is the shear viscosity, and $\delta_{i k}$ are Kronecker deltas. The average $\langle\cdots\rangle$ in Eq. (2) is over thermal noise, which is assumed to be a Markovian Gaussian stochastic process [1].

To solve Eq. (1) for the velocity fluctuations in equilibrium, we first eliminate the pressure term by taking a double curl of the linearized fluctuating Navier-Stokes equation in Eq. (1) and obtain as usual [41]

$$
\frac{\partial\left(\nabla^{2} \delta \mathbf{v}\right)}{\partial t}=\nu \nabla^{4} \delta \mathbf{v}+\mathbf{F}
$$

with a Langevin random noise term

$$
\mathbf{F}(\mathbf{r}, t)=\frac{1}{\rho_{0}} \nabla \times \nabla \times\{\nabla[\delta \Pi(\mathbf{r}, t)]\} .
$$

Equation (3) illustrates the well-known observation that the incompressibility approximation decouples the equation of the velocity fluctuations from any of the other fluctuatinghydrodynamics equations. Physically it means that in an incompressible liquid the pressure fluctuations are completely determined once the velocity fluctuations have been obtained from the solution of Eq. (3).

To solve Eq. (3) for the velocity fluctuations, we apply a full spatiotemporal Fourier transformation. In equilibrium the correlation fluctuations are short ranged and isotropic, independent of boundary conditions in any of the spatial directions. The random force $\mathbf{F}(\mathbf{r}, t)$ in Eqs. (3) and (4) represents a Langevin random noise term and the components of its temporal and spatial Fourier transform $\mathbf{F}(\omega, \mathbf{q})$ are given by

$$
F_{i}(\omega, \mathbf{q})=-\frac{i q_{j} q_{l} q_{s}}{\rho_{0}} \epsilon_{i j k} \epsilon_{k l m} \delta \Pi_{m s}(\omega, \mathbf{q}),
$$

where $\epsilon_{i j k}$ represent the components of the totally antisymmetric tensor, and summation over repeated indices is understood. The statistical correlations among the different components $F_{i}(\omega, \mathbf{q})$ of the random force $\mathbf{F}(\omega, \mathbf{q})$ can be readily calculated by combining Eq. (5) with the correlation functions among the various components of $\delta \Pi(\omega, \mathbf{q})$ deduced from Eq. (2). After some algebra one obtains

$$
\begin{aligned}
& \left\langle F_{i}(\omega, \mathbf{q}) \cdot F_{j}^{*}\left(\omega^{\prime}, \mathbf{q}^{\prime}\right)\right\rangle \\
& \quad=\frac{2 k_{B} T}{\rho_{0}} \nu q^{2}\left[q^{2} \delta_{i j}-q_{i} q_{j}\right](2 \pi)^{4} \delta\left(\omega-\omega^{\prime}\right) \delta\left(\mathbf{q}-\mathbf{q}^{\prime}\right) .
\end{aligned}
$$

Now we have all the information needed to obtain the elements $C_{i j}(\omega, \mathbf{q})$ of the correlation matrix $\mathbf{C}(\omega, \mathbf{q})$ for the velocity fluctuations, defined by

$$
\left\langle\delta v_{i}(\omega, \mathbf{q}) \cdot \delta v_{j}^{*}\left(\omega^{\prime}, \mathbf{q}^{\prime}\right)\right\rangle=C_{i j}(\omega, \mathbf{q})(2 \pi)^{4} \delta\left(\omega-\omega^{\prime}\right) \delta\left(\mathbf{q}-\mathbf{q}^{\prime}\right),
$$

where the presence of the two delta functions is expected, because of translational symmetry in space and time that exists in equilibrium. Applying a spatiotemporal Fourier transformation to Eq. (3), solving for the components of $\delta \mathbf{v}(\omega, \mathbf{q})$, and making use of Eq. (6) we obtain the following expression for $C_{i j}(\omega, \mathbf{q})$ :

$$
C_{i j}(\omega, \mathbf{q})=\left(\delta_{i j}-\hat{q}_{i} \hat{q}_{j}\right) \frac{k_{B} T}{\rho_{0}} \frac{2 \nu q^{2}}{\omega^{2}+\nu^{2} q^{4}},
$$

where $\hat{q}_{i}=q_{i} / q$. Notice that for an incompressible liquid all elements of the autocorrelation matrix for the velocity fluctuations are related to a single transverse-velocity-correlation function $C_{\perp}(\omega, \mathbf{q})$, such that 


$$
C_{i j}(\omega, \mathbf{q})=\left(\delta_{i j}-\hat{q}_{i} \hat{q}_{j}\right) C_{\perp}(\omega, \mathbf{q}) .
$$

In a compressible Newtonian fluid there are two independent elements of the correlation matrix for the velocity fluctuations, namely, transverse-velocity autocorrelation function (9) and a longitudinal-velocity autocorrelation function $[42,43]$. The incompressibility condition $\boldsymbol{\nabla} \cdot \delta \mathbf{v}=0$ causes the longitudinal-velocity autocorrelation function to vanish.

From the velocity fluctuations one readily obtains the well-known expression for the thermal kinetic energy. If we define an "average" velocity fluctuation in a given volume $V$ as

$$
\overline{\delta \mathbf{v}}(t)=\frac{1}{V} \int_{V} \delta \mathbf{v}(\mathbf{r}, t) d \mathbf{r},
$$

we have for the mean squared value of these velocity fluctuations

$$
\left\langle[\overline{\delta \mathbf{v}}(t)]^{2}\right\rangle=\frac{1}{V^{2}} \iint_{V \times V}\left\langle\delta v_{i}(\mathbf{r}, t) \delta v_{i}\left(\mathbf{r}^{\prime}, t\right)\right\rangle d \mathbf{r} d \mathbf{r}^{\prime} .
$$

If we apply an inverse Fourier transformation to Eq. (7) for the velocity-correlation tensor, substitute the result into Eq. (11), and perform the integrals over the spatial variables $\mathbf{r}$ and $\mathbf{r}^{\prime}$, we obtain

$$
\left\langle[\overline{\delta \mathbf{v}}(t)]^{2}\right\rangle=2 \frac{k_{B} T}{N m_{0}}=\left\langle v_{0}^{2}\right\rangle,
$$

where $N$ is the number molecules in a volume $V$ and $m_{0}$ is the molecular mass, so that $\rho_{0}=N m_{0} / V$. We thus reproduce a well-known result from statistical mechanics, referred to as the first sum rule of the velocity-correlation function $[42,43]$. Note that we obtain here a factor 2 on the right-hand side (RHS) of Eq. (12) (instead of the common 3) as a consequence of the incompressibility condition. Since $\boldsymbol{\nabla} \cdot \delta \mathbf{v}=0$, only two components of the velocity can fluctuate independently. For compressible fluids the longitudinal-velocity autocorrelation function is not zero and instead of Eq. (12) one would obtain the regular result (i.e., $\left\langle\delta \epsilon_{\text {kin }}\right\rangle=\frac{1}{2} N m_{0}\left\langle v_{0}^{2}\right\rangle$ $\left.=\frac{3}{2} k_{B} T\right)[42,43]$.

\section{STOCHASTIC ORR-SOMMERFELD AND SQUIRE EQUATIONS}

In this section we consider an isothermal liquid under incompressible flow (thus, with uniform density $\rho$ ) that is located between two horizontal plates separated by a distance $2 L$. The mean flow velocity $\mathbf{v}_{0}=\{\dot{\gamma} z, 0,0\}$ is in the $x$ direction, with $\dot{\gamma}$ representing a constant shear rate in the $z$ direction. We continue to use here the coordinate system adopted in our previous publication that agrees with the nomenclature of Drazin and Reid [20] and which is sometimes referred to as the meteorological convention [34]. Thus our $x, y$, and $z$ directions correspond to what are being designated in the fluid-dynamics literature as the streamwise, spanwise, and (wall-) normal directions, respectively [32,21]. This physical situation is usually referred to as planar or plane Couette flow.
It is convenient to introduce dimensionless variables, defined by

$$
\begin{gathered}
\tilde{\mathbf{r}}=\frac{\mathbf{r}}{L}, \quad \tilde{t}=\dot{\gamma} t, \quad \delta \widetilde{p}=\frac{\delta p}{\rho_{0} L^{2} \dot{\gamma}^{2}}, \\
\delta \widetilde{\mathbf{v}}=\frac{\delta \mathbf{v}}{L \dot{\gamma}}, \quad \delta \widetilde{\Pi}=\frac{\delta \Pi}{\rho_{0} L^{2} \dot{\gamma}^{2}}, \quad \tilde{q}=q L, \quad \widetilde{\omega}=\omega / \dot{\gamma} .
\end{gathered}
$$

In a previous publication [36] we have shown how, starting from the fluctuating-hydrodynamics equations for incompressible flow, one can eliminate the pressure fluctuations in a way similar to the derivation of Eq. (3) and obtain two dimensionless fluctuating-hydrodynamics equations for the fluctuations $\delta v_{z}(\mathbf{r}, t)$ and $\delta \omega_{z}=\partial_{y} \delta v_{x}-\partial_{x} \delta v_{y}$ of the normal components of the velocity and vorticity, respectively,

$$
\partial_{t}\left(\nabla^{2} \delta v_{z}\right)+z \partial_{x}\left(\nabla^{2} \delta v_{z}\right)-\frac{1}{\operatorname{Re}} \nabla^{4}\left(\delta v_{z}\right)=F_{z}
$$

and

$$
\partial_{t}\left(\delta \omega_{z}\right)+z \partial_{x}\left(\delta \omega_{z}\right)-\partial_{y} \delta v_{z}-\frac{1}{\operatorname{Re}} \nabla^{2}\left(\delta \omega_{z}\right)=R_{z},
$$

where

$$
\operatorname{Re}=\dot{\gamma} L^{2} / \nu
$$

is the Reynolds number, $F_{z}(\mathbf{r}, t)$ in Eq. (14) is the dimensionless wall-normal component of the same random force $\mathbf{F}(\mathbf{r}, t)$ appearing in the RHS of Eq. (3), while Eq. (15) has a thermal noise term $R_{z}(\mathbf{r}, t)$ that is the wall-normal component of

$$
\mathbf{R}(\mathbf{r}, t)=\boldsymbol{\nabla} \times\{\boldsymbol{\nabla}[\delta \Pi(\mathbf{r}, t)]\} .
$$

Notice that in Eqs. (14) and (15) we have omitted the tildes in denoting the variables since from now on we shall exclusively use dimensionless quantities (except in some explicitly indicated places in Sec. VI). Equations (14) and (15) form a pair of coupled stochastic differential equations, which need to be solved for the fluctuating fields $\delta v_{z}(\mathbf{r}, t)$ and $\delta \omega_{z}(\mathbf{r}, t)$ with appropriate boundary conditions. The deterministic versions of Eqs. (14) and (15) are known as the Orr-Sommerfeld equation and the Squire equation, respectively $[20,21]$. Our basic assumption is that in the presence of flow we can continue to use the same expressions for the correlation functions between the components of the random stress tensor $\delta \Pi(\mathbf{r}, t)$ on the RHS of the Orr-Sommerfeld and Squire equations [Eqs. (14) and (15)] as in the absence of flow, for which the autocorrelation function of the random stress tensor $\delta \Pi(\mathbf{r}, t)$ is given by Eq. (2) according to the well-known fluctuating-dissipation theorem. One important consequence of this assumption is that the random noise term in the stochastic Orr-Sommerfeld equation is uncorrelated with that in the Squire equation, namely,

$$
\left\langle F_{z}^{*}(\mathbf{r}, t) \cdot R_{z}\left(\mathbf{r}^{\prime}, t^{\prime}\right)\right\rangle=\left\langle R_{z}^{*}(\mathbf{r}, t) \cdot F_{z}\left(\mathbf{r}^{\prime}, t^{\prime}\right)\right\rangle=0,
$$

while the autocorrelations of $F_{z}(\mathbf{r}, t)$ and $R_{z}(\mathbf{r}, t)$ will be discussed later. The extension of fluctuating hydrodynamics to nonequilibrium states by applying a local version of the fluctuation-dissipation theorem has been verified experimen- 
tally for fluctuations in liquids with a temperature gradient and in liquid mixtures with a concentration gradient [1].

As shown in a previous publication, the stochastic OrrSommerfeld equation [Eq. (14)] predicts a nonequilibrium enhancement of the intensity of the normal-velocity fluctuations that depends on the value of the Reynolds number [36]. This nonequilibrium enhancement is caused by a coupling of the same modes (associated with the normal-velocity fluctuations) but with different wave numbers. We shall refer to this mechanism as self-coupling. As a consequence the nonequilibrium enhancement of the normal-velocity fluctuations can be described by a single partial differential equation [Eq. (14)]. However, unlike the Orr-Sommerfeld equation, the Squire equation also includes a coupling of the vorticity fluctuations with the velocity fluctuations (with the same wave number), besides the self-coupling mechanism. Hence, to determine the nonequilibrium enhancement of the normalvorticity fluctuations we need to solve the two coupled stochastic equations [Eqs. (14) and (15)] simultaneously.

\section{NONEQUILIBRIUM VELOCITY FLUCTUATIONS IN THE ABSENCE OF BOUNDARY CONDITIONS}

The solutions of the stochastic Orr-Sommerfeld and Squire equations should satisfy appropriate boundary conditions for $\delta v_{z}$ and $\delta \omega_{z}$ at the two horizontal planes. Here we first consider the solutions in the absence of any boundary conditions. The idea is that the resulting solutions are valid inside the bulk of the liquid at wavelengths much smaller than the distance between the plates.

The solution of the stochastic Orr-Sommerfeld equation [Eq. (14)] was presented in a previous publication [36]. There it was shown that the solution in the absence of any boundary conditions can be obtained by applying a full spatiotemporal Fourier transformation to Eq. (14),

$$
q^{2}\left[i \omega+\frac{q^{2}}{\operatorname{Re}}\right] \delta v_{z}(\omega, \mathbf{q})-q_{x} \frac{\partial}{\partial q_{z}}\left[q^{2} \delta v_{z}(\omega, \mathbf{q})\right]=F_{z}(\omega, \mathbf{q}) .
$$

To solve Eq. (19) for $\delta v_{z}(\omega, \mathbf{q})$ we followed Lutsko and Dufty $[37,44,45]$ by expressing the solution in terms of a Green's function, namely,

$$
\delta v_{z}(\omega, \mathbf{q})=\int_{-\infty}^{\infty} d q_{z}^{\prime} \frac{G\left(\omega, \mathbf{q}_{\|}, q_{z}, q_{z}^{\prime}\right)}{q_{\|}^{2}+q_{z}^{2}} F_{z}\left(\omega, \mathbf{q}_{\|}, q_{z}^{\prime}\right),
$$

where the Green's function $G\left(\omega, \mathbf{q}_{\|}, q_{z}, q_{z}^{\prime}\right)$ is the solution of $\left[i \omega+\frac{q^{2}}{\operatorname{Re}}\right] G\left(\omega, \mathbf{q}_{\|}, q_{z}, q_{z}^{\prime}\right)-q_{x} \frac{\partial}{\partial q_{z}} G\left(\omega, \mathbf{q}_{\|}, q_{z}, q_{z}^{\prime}\right)=\delta\left(q_{z}-q_{z}^{\prime}\right)$.

In Eqs. (20) and (21), $\mathbf{q}_{\|}=\left\{q_{x}, q_{y}\right\}$ represents the projection of the fluctuations wave vector $\mathbf{q}$ onto the horizontal plane, parallel to the fluid boundaries. Please note that to shorten the notation we have slightly modified the definition of the Green's function $G\left(\omega, \mathbf{q}_{\|}, q_{z}, q_{z}^{\prime}\right)$ as compared to that used in the previous publication [36] by incorporating a factor $q^{2}$.

We now solve the stochastic Squire equation [Eq. (15)] by a similar procedure. Thus we first apply a full spatiotemporal
Fourier transformation to Eq. (15), so as to obtain

$$
\begin{gathered}
{\left[i \omega+\frac{q^{2}}{\operatorname{Re}}\right] \delta \omega_{z}(\omega, \mathbf{q})-q_{x} \frac{\partial}{\partial q_{z}} \delta \omega_{z}(\omega, \mathbf{q})} \\
=R_{z}(\omega, \mathbf{q})+i q_{y} \delta v_{z}(\omega, \mathbf{q})
\end{gathered}
$$

where $R_{z}(\omega, \mathbf{q})$ is the wall-normal component of the spatiotemporal Fourier transform of the Langevin random-force term, defined by Eq. (17) in terms of the random stress,

$$
R_{z}(\omega, \mathbf{q})=q_{i} q_{j} \epsilon_{z j i} \delta \Pi_{i j}(\omega, \mathbf{q}) .
$$

We observe that the solution $\delta \omega_{z}(\omega, \mathbf{q})$ of the (Fouriertransformed) Squire equation [Eq. (22)] can be expressed in terms of the Green's function $G\left(\omega, \mathbf{q}_{\|}, q_{z}, q_{z}^{\prime}\right)$ and an extra contribution $\delta \omega_{z}^{(2)}(\omega, \mathbf{q})$, such that

$$
\delta \omega_{z}(\omega, \mathbf{q})=\int_{-\infty}^{\infty} d q_{z}^{\prime} G\left(\omega, \mathbf{q}_{\|}, q_{z}, q_{z}^{\prime}\right) R_{z}\left(\omega, \mathbf{q}_{\|}, q_{z}^{\prime}\right)+\delta \omega_{z}^{(2)}(\omega, \mathbf{q})
$$

where the function $\delta \omega_{z}^{(2)}(\omega, \mathbf{q})$ is the solution of

$$
\left[i \omega+\frac{q^{2}}{\operatorname{Re}}\right] \delta \omega_{z}^{(2)}-q_{x} \frac{\partial}{\partial q_{z}} \delta \omega_{z}^{(2)}=i q_{y} \delta v_{z}
$$

with $\delta v_{z}(\omega, \mathbf{q})$ given by Eq. (20) and, hence, being the solution of the stochastic Orr-Sommerfeld equation.

Notice that in Eq. (24) $\delta \omega_{z}^{(2)}(\omega, \mathbf{q})$ is a random function, while the Green's function $G\left(\omega, \mathbf{q}_{\|}, q_{z}, q_{z}^{\prime}\right)$ is a deterministic function. Representation (24) for the solution of the stochastic Squire equation as a sum of two terms reflects the presence of two distinct coupling mechanisms. The first term on the RHS of Eq. (24) is similar to expression (20) for the solution of the stochastic Orr-Sommerfeld equation and represents the result of the self-coupling mechanism between vorticity fluctuations with different $q_{z}$. This mode-coupling mechanism is also present in the stochastic Squire equation. However, the second term on the RHS of Eq. (24) accounts for a mode coupling between the velocity and vorticity fluctuations in the Squire equation that is not present in the OrrSommerfeld equation.

To determine the autocorrelation functions $\left\langle\delta v_{z}^{*}(\omega, \mathbf{q}) \cdot \delta v_{z}\left(\omega^{\prime}, \mathbf{q}^{\prime}\right)\right\rangle$ and $\left\langle\delta \omega_{z}^{*}(\omega, \mathbf{q}) \cdot \delta \omega_{z}\left(\omega^{\prime}, \mathbf{q}^{\prime}\right)\right\rangle$, we need the correlation functions between the random noise terms on the RHS of the Fourier-transformed stochastic OrrSommerfeld and Squire equations [Eqs. (19) and (22)]. These correlation functions can be readily evaluated from Eq. (2) for the elements of the random stress tensor $\delta \Pi$. From the definition of $F_{z}(\omega, \mathbf{q})$ in Eq. (5) and of $R_{z}(\omega, \mathbf{q})$ in Eq. (23), one then obtains

$$
\frac{\left\langle F_{z}^{*}(\omega, \mathbf{q}) \cdot F_{z}\left(\omega^{\prime}, \mathbf{q}^{\prime}\right)\right\rangle}{2 \tilde{S}(2 \pi)^{4}}=q_{\|}^{2} q^{4} \delta\left(\omega-\omega^{\prime}\right) \delta\left(\mathbf{q}-\mathbf{q}^{\prime}\right),
$$




$$
\frac{\left\langle R_{z}^{*}(\omega, \mathbf{q}) \cdot R_{z}\left(\omega^{\prime}, \mathbf{q}^{\prime}\right)\right\rangle}{2 \widetilde{S}(2 \pi)^{4}}=q_{\|}^{2} q^{2} \delta\left(\omega-\omega^{\prime}\right) \delta\left(\mathbf{q}-\mathbf{q}^{\prime}\right),
$$

while the cross-correlation $\left\langle F_{z}^{*}(\omega, \mathbf{q}) \cdot R_{z}\left(\omega^{\prime}, \mathbf{q}^{\prime}\right)\right\rangle$ is zero as a consequence of Eq. (18). In Eq. (25) we have introduced a dimensionless strength $\widetilde{S}$ of the thermal noise given by [36]

$$
\tilde{S}=\frac{k_{B} T}{\dot{\gamma}^{3} L^{7}} \frac{\nu}{\rho_{0}}=\frac{k_{B} T}{\rho_{0} L^{3}} \frac{1}{\dot{\gamma}^{2} L^{2}} \frac{1}{\operatorname{Re}} .
$$

We note again that dimensionless quantities (including a dimensionless random stress) are used in this paper.

\section{A. Solution procedure}

Here we explicitly solve Eqs. (21) and (25) for the Green's function $G\left(\omega, \mathbf{q}_{\|}, q_{z}, u\right)$ and the contribution $\delta \omega_{z}^{(2)}(\omega, \mathbf{q})$ to the vorticity fluctuations. The solutions can be formally expressed as

$$
\begin{aligned}
& G\left(q_{z}, q_{z}^{\prime}\right)=\int_{0}^{\infty} e^{-\Omega\left(q_{z}, \alpha\right)} \delta\left(q_{z}-q_{z}^{\prime}+q_{x} \alpha\right) d \alpha, \\
& \delta \omega_{z}^{(2)}\left(q_{z}\right)=i q_{y} \int_{0}^{\infty} e^{-\Omega\left(q_{z}, \alpha\right)} \delta v_{z}\left(q_{z}+q_{x} \alpha\right) d \alpha,
\end{aligned}
$$

with

$$
\Omega\left(q_{z}, \alpha\right)=\frac{q_{x}^{2}}{3 \operatorname{Re}} \alpha^{3}+\frac{q_{x} q_{z}}{\operatorname{Re}} \alpha^{2}+\left[i \omega+\frac{q_{\|}^{2}+q_{z}^{2}}{\operatorname{Re}}\right] \alpha .
$$

To simplify the notation, we dropped the implicit $\omega, \mathbf{q}_{\|}$dependence of all quantities appearing in Eqs. (28)-(30), retaining explicitly only the $q_{z}$ dependence of $\delta \omega_{z}^{(2)}$, and the dependence on $q_{z}$ and $q_{z}^{\prime}$ of the Green's function. Notice that Eqs. (29) and (30) are valid for both positive and negative values of $q_{x}$.

Upon substituting Eq. (20) for $\delta v_{z}(\omega, \mathbf{q})$ into Eq. (29) for $\delta \omega_{z}^{(2)}(\omega, \mathbf{q})$ and using explicit expression (28) of the Green's function, we obtain an alternative expression that will turn out to be more useful in the following. Specifically,

$$
\begin{aligned}
\delta \omega_{z}^{(2)}\left(q_{z}\right)= & i q_{y} \int_{-\infty}^{\infty} d u F_{z}(u) \int_{0}^{\infty} \int_{0}^{\infty} d \alpha d \beta \frac{\exp \left[-\Omega\left(q_{z}, \alpha+\beta\right)\right]}{q_{\|}^{2}+\left(q_{z}+q_{x} \beta\right)^{2}} \\
& \times \delta\left(q_{z}-u+q_{x}(\alpha+\beta)\right),
\end{aligned}
$$

which can be simplified by integration over polar coordinates in the first quadrant of the $\{\alpha, \beta\}$ plane into

$$
\begin{aligned}
\delta \omega_{z}^{(2)}\left(q_{z}\right)= & 2 i q_{y} \int_{-\infty}^{\infty} d u F_{z}(u) \int_{0}^{\infty} d \alpha \int_{0}^{1} d s \frac{\alpha s \exp \left[-\Omega\left(q_{z}, \alpha\right)\right]}{q_{\|}^{2}+\left(q_{z}+q_{x} \alpha s^{2}\right)^{2}} \\
& \times \delta\left(q_{z}-u+q_{x} \alpha\right) .
\end{aligned}
$$

The integral over the auxiliary variable $s$ can be performed explicitly.

\section{B. Correlation functions of wall-normal-velocity and vorticity fluctuations}

Our ultimate goal is to evaluate the intrinsic nonequilibrium energy amplification, that is, the energy amplification in the absence of any externally imposed noise correlations. For this purpose we need the correlation functions of the vertical components $\delta v_{z}$ and $\delta \omega_{z}$ of the velocity and vorticity fluctuations.

The correlation function of the fluctuations of the wallnormal component $\delta v_{z}$ of the velocity, previously referred to as transverse velocity, was derived in our previous publication [36]. The resulting expression was shown to have the form

$$
\begin{aligned}
& \left\langle\delta v_{z}^{*}(\omega, \mathbf{q}) \cdot \delta v_{z}\left(\omega^{\prime}, \mathbf{q}^{\prime}\right)\right\rangle \\
& \quad=C_{z z}\left(\omega, \mathbf{q}_{\|}, q_{z}, q_{z}^{\prime}\right)(2 \pi)^{3} \delta\left(\omega-\omega^{\prime}\right) \delta\left(\mathbf{q}_{\|}-\mathbf{q}_{\|}^{\prime}\right)
\end{aligned}
$$

with the function $C_{z z}\left(\omega, \mathbf{q}_{\|}, q_{z}, q_{z}^{\prime}\right)$ given by Eq. (20) in Ref. [36]. As is to be expected from translational time invariance of the steady state, the autocorrelation function of the transverse-velocity fluctuations is proportional to $\delta\left(\omega-\omega^{\prime}\right)$. Because of translational invariance in the horizontal $x y$ plane, it is also proportional to $\delta\left(\mathbf{q}_{\|}-\mathbf{q}_{\|}^{\prime}\right)$. However, as was discussed in more detail in Ref. [36], this correlation function is not invariant under translations in the vertical direction.

Here we proceed with the evaluation of the correlation function $\left\langle\delta \omega_{z}^{*}(\omega, \mathbf{q}) \cdot \delta \omega_{z}\left(\omega^{\prime}, \mathbf{q}^{\prime}\right)\right.$ for the wall-normalvorticity fluctuations. From representation (24) of $\delta \omega_{z}(\omega, \mathbf{q})$ we conclude that this autocorrelation function will contain two distinct contributions. The first contribution is the autocorrelation of the first term in the RHS of Eq. (24), which arises from the self-coupling of the vorticity fluctuations. The second contribution is $\left\langle\delta \omega_{z}^{(2) *}(\omega, \mathbf{q}) \delta \omega_{z}^{(2)}\left(\omega^{\prime}, \mathbf{q}^{\prime}\right)\right\rangle$ which arises from the coupling of the vorticity fluctuations and the velocity fluctuations through Eq. (25). Because of the structure of thermal noise (18), the cross correlation between the first and the second contributions in the RHS of Eq. (24) vanishes. In addition, from Eq. (25) it follows that each one of the two nonzero contributions will be proportional to delta functions in frequency and in the $x y$ component of the wave number. Therefore, we find that similarly to Eq. (33),

$$
\begin{aligned}
& \left\langle\delta \omega_{z}^{*}(\omega, \mathbf{q}) \cdot \delta \omega_{z}\left(\omega^{\prime}, \mathbf{q}^{\prime}\right)\right\rangle \\
& \quad=W_{z z}\left(\omega, \mathbf{q}_{\|}, q_{z}, q_{z}^{\prime}\right)(2 \pi)^{3} \delta\left(\omega-\omega^{\prime}\right) \delta\left(\mathbf{q}_{\|}-\mathbf{q}_{\|}^{\prime}\right),
\end{aligned}
$$

where the function $W_{z z}$ is also the sum of two contributions corresponding to the two coupling mechanisms mentioned above. Explicit expressions for each one of these two contributions can be readily obtained by combining Eqs. (29) and (32) with the correlation functions [Eq. (25)] between the two components of the thermal noise. Here we do not display the expressions for $W_{z z}$ explicitly. First, the explicit expressions are very long and not informative. Second, for an evaluation of the energy amplification we only need the equal-time-correlation functions. For these reasons we do not discuss here the frequency spectrum of the vorticity fluctuations, a phenomenon that is complicated due to the nontranslational invariance along the wall-normal direction 
The equal-time autocorrelation functions of the wallnormal-velocity and vorticity fluctuations are ready obtained by applying a double Fourier (anti)transform to Eqs. (33) and (34) with the results

$$
\begin{aligned}
& \frac{\left\langle\delta v_{z}^{*}(\mathbf{q}, t) \cdot \delta v_{z}\left(\mathbf{q}^{\prime}, t\right)\right\rangle}{(2 \pi)^{2} \delta\left(\mathbf{q}_{\|}-\mathbf{q}_{\|}^{\prime}\right)}=\frac{1}{2 \pi} \int_{-\infty}^{\infty} C_{z z}\left(\omega, \mathbf{q}_{\|}, q_{z}, q_{z}^{\prime}\right) d \omega, \\
& \frac{\left\langle\delta \omega_{z}^{*}(\mathbf{q}, t) \cdot \delta \omega_{z}\left(\mathbf{q}^{\prime}, t\right)\right\rangle}{(2 \pi)^{2} \delta\left(\mathbf{q}_{\|}-\mathbf{q}_{\|}^{\prime}\right)}=\frac{1}{2 \pi} \int_{-\infty}^{\infty} W_{z z}\left(\omega, \mathbf{q}_{\|}, q_{z}, q_{z}^{\prime}\right) d \omega .
\end{aligned}
$$

The integration over frequency of $C_{z z}\left(\omega, \mathbf{q}_{\|}, q_{z}, q_{z}^{\prime}\right)$ for the wall-normal velocity fluctuations was evaluated in our preceding publication on the subject [36], where we showed that

$$
\int_{-\infty}^{\infty} d \omega C_{z z}\left(\omega, \mathbf{q}_{\|}, q_{z}, q_{z}^{\prime}\right)=C_{z z}(\mathbf{q}) 4 \pi^{2} \delta\left(q_{z}-q_{z}^{\prime}\right)
$$

with the intensity $C_{z z}(\mathbf{q})$ of the wall-normal-velocity fluctuations splitting additively into an equilibrium and a nonequilibrium contribution, namely,

$$
C_{z z}(\mathbf{q})=C_{z z}^{(\mathrm{E})}(\mathbf{q})+C_{z z}^{(\mathrm{NE})}(\mathbf{q})
$$

with

$$
\begin{gathered}
C_{z z}^{(\mathrm{E})}(\mathbf{q})=\tilde{S} \operatorname{Re} \frac{q_{\|}^{2}}{q^{2}}, \\
C_{z z}^{(\mathrm{NE})}(\mathbf{q})=2 \tilde{S} \operatorname{Re} \frac{q_{x} q_{\|}^{2}}{q^{4}} \int_{0}^{\infty} d \beta\left(q_{z}+q_{x} \beta\right) e^{-\Gamma(\beta, \mathbf{q})},
\end{gathered}
$$

and

$$
\Gamma(\beta, \mathbf{q})=\frac{2 \beta}{3 \operatorname{Re}}\left(q_{x}^{2} \beta^{2}+3 \beta q_{x} q_{z}+3 q^{2}\right) .
$$

Here we perform a completely similar calculation, but now for the wall-normal-vorticity fluctuations obtained by solving the stochastic Squire equation [Eq. (15)]. Integration of the explicit expression for $W_{z z}\left(\omega, \mathbf{q}_{\|}, q_{z}, q_{z}^{\prime}\right)$ over the frequency $\omega$ yields, similarly to Eq. (37),

$$
\int_{-\infty}^{\infty} d \omega W_{z z}\left(\omega, \mathbf{q}_{\|}, q_{z}, q_{z}^{\prime}\right)=W_{z z}(\mathbf{q}) 4 \pi^{2} \delta\left(q_{z}-q_{z}^{\prime}\right) .
$$

Note that Eq. (41), as well as Eq. (37), demonstrates the translational invariance of the equal-time velocity and vorticity fluctuations when boundary conditions are not taken into account. Hence, although the temporal spectrum of these fluctuations does not satisfy translational invariance, the total intensity of the fluctuations is translational invariant

Of course, the intensity $W_{z z}(\mathbf{q})$ of the wall-normalvorticity fluctuations will inherit the two contributions to $W_{z z}(\omega, \mathbf{q})$ corresponding to the two distinct coupling mechanisms mentioned after Eqs. (32) and (34). Specifically we find

$$
W_{z z}(\mathbf{q})=W_{z z}^{(\mathrm{E})}(\mathbf{q})+W_{z z}^{(\mathrm{NE})}(\mathbf{q})
$$

with

$$
W_{z z}^{(\mathrm{E})}(\mathbf{q})=2 \widetilde{S} q_{\|}^{2} \int_{0}^{\infty} d \beta\left[q_{\|}^{2}+\left(q_{z}+q_{x} \beta\right)^{2}\right] e^{-\Gamma(\beta, \mathbf{q})}=\widetilde{S} \operatorname{Re} q_{\|}^{2},
$$

$$
\begin{aligned}
W_{z z}^{(\mathrm{NE})}(\mathbf{q})= & 2 \widetilde{S} \frac{q_{y}^{2}}{q_{x}^{2}} \int_{0}^{\infty} d \beta\left[q_{\|}^{2}+\left(q_{z}+q_{x} \beta\right)^{2}\right]^{2} \\
& \times\left[\arctan \left(\frac{q_{z}+q_{x} \beta}{q_{\|}}\right)-\arctan \left(\frac{q_{z}}{q_{\|}}\right)\right]^{2} e^{-\Gamma(\beta, \mathbf{q})} .
\end{aligned}
$$

Recalling that the coefficient $\tilde{S}$, given by Eq. (27), is inversely proportional to the Reynolds number Re, we note that the first contribution to $W_{z z}(\mathbf{q})$, given by Eq. (43a) and arising from the autocorrelation of the first term in the RHS of Eq. (24), is independent of the Reynolds number and reproduces the equilibrium result for the autocorrelation of the vertical vorticity fluctuations. We thus arrive at the interesting conclusion that the self-coupling of the vorticity fluctuations does not yield any nonequilibrium contribution to the intensity of the vorticity fluctuations. Only the second contribution to $W_{z z}(\mathbf{q})$, given by Eq. (43b) and arising from the coupling of the vorticity fluctuations and the velocity fluctuations through $\delta \omega_{z}^{(2)}(\omega, \mathbf{q})$ in Eq. (24), yields a contribution to a nonequilibrium intensity of the vorticity fluctuations. For this reason we have used the superscripts (E) and (NE) to distinguish between these two contributions to the wallnormal-vorticity correlation function. It can be easily verified that the nonequilibrium contribution $W_{z z}^{(\mathrm{NE})}(\mathbf{q})$ from the selfcoupling of the vorticity fluctuations varies for small value of the Reynolds number as $\mathrm{Re}^{2}$.

Regarding the anisotropic nature of the fluctuations we note that the intensity, $C_{z z}^{(\mathrm{NE})}(\mathbf{q})$, of the nonequilibrium wallnormal-velocity fluctuations vanishes at $q_{x}=0$ and has a maximum at $q_{y}=0$. On the other hand, the intensity of the nonequilibrium wall-normal-vorticity fluctuations, $W_{z z}^{(\mathrm{NE})}(\mathbf{q})$, vanishes at $q_{y}=0$ and has a maximum at $q_{x}=0$. We are not able to evaluate integral (43b) for the intensity of the nonequilibrium vorticity fluctuations analytically so as to specify its dependence on the wave vector $\mathbf{q}$ explicitly. However, it is possible to obtain the asymptotic expansions for large and small wave numbers $q$. To specify these limits we introduce spherical coordinates by substituting $q_{z}=q \cos \theta, q_{\|}=q \sin \theta$ and $q_{x}=q \sin \theta \cos \phi$ into Eq. (43b). After these substitutions, the two above mentioned limits can be properly taken, and we obtain

$$
\begin{aligned}
W_{z z}^{(\mathrm{NE})}(\mathbf{q}) \stackrel{q \rightarrow 0}{\rightarrow} & \widetilde{S}\left(\frac{2}{3}\right)^{1 / 3} \Gamma\left(\frac{2}{3}\right) \operatorname{Re}^{5 / 3}(\cos \phi \sin \theta)^{2 / 3} \\
& \times(\theta \tan \phi)^{2} q^{2 / 3},
\end{aligned}
$$




$$
W_{z z}^{(\mathrm{NE})}(\mathbf{q}) \stackrel{q \rightarrow \infty}{\rightarrow} \widetilde{S} \frac{\operatorname{Re}^{3}}{2 q^{2}} \sin ^{4} \theta \sin ^{2} \phi,
$$

where the small- $q$ expansion is only valid for $\cos \phi>0$. From Eq. (44) we conclude that the nonequilibrium enhancement of the normal-vorticity fluctuations (i.e., the ratio of the intensities of the nonequilibrium and equilibrium fluctuations) exhibits a crossover from a $q^{2 / 3}$ dependence for small $q$ to a $q^{-2}$ dependence for large $q$. In a previous publication [36] we have shown that the nonequilibrium enhancement of the normal-velocity fluctuations exhibits a crossover from a $q^{-4 / 3}$ dependence for smaller $q$ to a competition between a $q^{-2}$ and a $q^{-4}$ dependence for large $q$, depending on the direction of the wave vector $\mathbf{q}$. It should be noted that while the wave-number dependence for large $q$ is an intrinsic property of the fluctuations in the bulk of the liquid, the wavenumber dependence for small $q$ is strongly affected by the presence of boundaries, to be considered in Sec. V.

The asymptotic Eq. (44b) ceases to be valid for $\cos \phi=0$, i.e., for wave vectors in the plane in which the spanwise direction is located $\left(q_{x}=0\right)$. If we take the limit $q_{x} \rightarrow 0$ in Eq. (43b) and then perform the integration over the variable $\beta$ we obtain for $q_{x}=0$

$$
W_{z z}^{(\mathrm{NE})}\left(q_{x}=0, q_{y}, q_{z}\right)=\frac{1}{2} \widetilde{S} \frac{q_{y}^{4} \mathrm{Re}^{3}}{\left(q_{y}^{2}+q_{z}^{2}\right)^{3}},
$$

which is proportional to the cube of the Reynolds number.

Another limit worth investigating is $q_{z} \rightarrow 0(\sin \theta=1)$. In this case the wave vector $\mathbf{q}=\mathbf{q}_{\|}$is perpendicular to the $z$ components of the fluctuating velocity and vorticity, i.e., parallel to the two boundaries. For this reason we have referred to $\delta v_{z}$ at $q_{z}=0$ as transverse-velocity fluctuations in our previous publication [36]. As will be discussed in more detail in Sec. VI, taking the limit $q_{z} \rightarrow 0$ corresponds to averaging the fluctuations over the wall-normal direction. In Fig. 1 we show a plot of the normalized intensity $W_{z z}^{(\mathrm{NE})}\left(\mathbf{q}_{\|}\right) / \widetilde{S}$ of the nonequilibrium normal-vorticity fluctuations, deduced from Eq. (43b) as a function of the magnitude $q_{\|}$of the wave vector $\mathbf{q}_{\|}$for $q_{z}=0, q_{x}=q_{y}(\phi=\pi / 4)$, and $\operatorname{Re}=20$. The choice $\phi=\pi / 4$ represents a value intermediate between $\phi=0\left(q_{y}=0\right)$ for which $W_{z z}^{(\mathrm{NE})}$ vanishes and $\phi=\pi / 2$ for which $W_{z z}^{(\mathrm{NE})}$ is given by Eq. (45) and the asymptotic algebraic $q^{2 / 3}$ at small $q$ vanishes. As is evident from Fig. 1, the intensity of the nonequilibrium normal-vorticity fluctuations is essentially a crossover between the two asymptotic behaviors described by Eq. (44).

\section{NONEQUILIBRIUM VELOCITY FLUCTUATIONS IN THE PRESENCE OF BOUNDARY CONDITIONS}

Planar Couette flow cannot be maintained without two bounding plates that drive the fluid flow. In our previous publication we showed that the presence of boundary conditions profoundly modifies the spatial spectrum of the velocity fluctuations [36]. Hence, we should expect that confinement will also affect the vorticity fluctuations. The appropriate boundary condition for the wall-normal-vorticity fluctuations at the two bounding plates is [41]

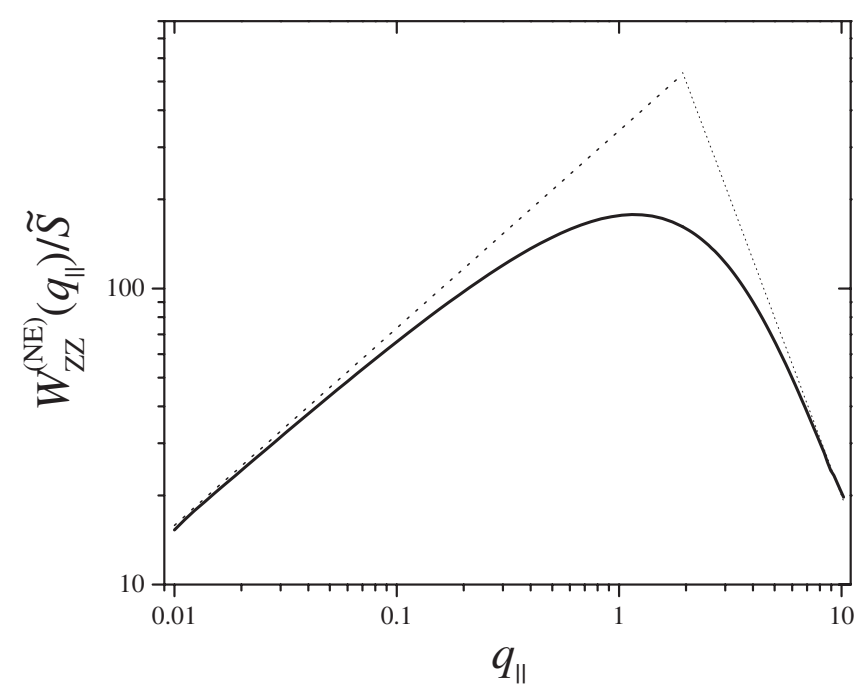

FIG. 1. Normalized intensity $W_{z z}^{(\mathrm{NE})}\left(\mathbf{q}_{\|}\right) / \tilde{S}$ of the nonequilibrium wall-normal-vorticity fluctuations as a function of the horizontal wave number $q_{\|}$for $q_{z}=0, q_{x}=q_{y}=q(\phi=\pi / 4)$ and $\operatorname{Re}=20$. The straight lines represent the asymptotic behaviors for small and large $q_{\|}$, as given by Eq. (44).

$$
\delta \omega_{z}=0 \quad \text { at } z= \pm 1 .
$$

We remind the reader that we continue to use dimensionless lengths scaled by the half-height of the liquid layer.

To accommodate the boundary conditions [Eq. (46)], we apply to the Squire equation [Eq. (15)] a spatiotemporal Fourier transformation, but restricting the spatial transformation to only the horizontal $x y$ plane. The resulting stochastic differential equation can be expressed as

$$
\mathcal{S}\left(\omega, \mathbf{q}_{\|}, z\right) \cdot \delta \omega_{z}\left(\omega, \mathbf{q}_{\|}, z\right)=F_{2}\left(\omega, \mathbf{q}_{\|}, z\right),
$$

where the Squire linear differential operator is

$$
\mathcal{S}\left(\omega, \mathbf{q}_{\|}, z\right)=i\left[\omega+z q_{x}\right]-\frac{1}{\operatorname{Re}}\left(\partial_{z}^{2}-q_{\|}^{2}\right),
$$

and the stochastic force

$$
F_{2}\left(\omega, \mathbf{q}_{\|}, z\right)=R_{z}\left(\omega, \mathbf{q}_{\|}, z\right)+i q_{y} \delta v_{z}\left(\omega, \mathbf{q}_{\|}, z\right),
$$

with $R_{z}\left(\omega, \mathbf{q}_{\|}, z\right)$ representing the Fourier transform of the vertical component of the random force $\mathbf{R}(\mathbf{r}, t)$, as defined by Eq. (17) in terms of the random stress. The physical significance of Eq. (47) is that the vertical-velocity fluctuations $\delta v_{z}\left(\omega, \mathbf{q}_{\|}, z\right)$, obtained from solving the Orr-Sommerfeld equation with proper boundary conditions, act here as an independent source of (additive) noise in the fluctuating Squire equation. Otherwise, the approach is similar to the one followed in Sec. IV for determining the vorticity fluctuations in the bulk without boundary conditions.

To obtain an analytic solution of the stochastic Squire equation [Eq. (47)] in a fluid layer of finite height we adopt a Galerkin approximation just as we did previously for the evaluation of the confinement effect on the wall-normalvelocity fluctuations [36]. Thus, we express the solution of Eq. (47) in terms of a series, 


$$
\delta \omega_{z}\left(\omega, \mathbf{q}_{\|}, z\right)=\left(z^{2}-1\right) \sum_{N=0}^{\infty} B_{N}\left(\omega, \mathbf{q}_{\|}\right) z^{N} .
$$

This expression for $\delta \omega_{z}\left(\omega, \mathbf{q}_{\|}, z\right)$ satisfies the boundary conditions [Eq. (46)] for any set of coefficients $B_{N}\left(\omega, \mathbf{q}_{\|}\right)$. The polynomial $\left(z^{2}-1\right)$ that forces $\delta \omega_{z}\left(\omega, \mathbf{q}_{\|}, z\right)$ to satisfy the boundary conditions is usually referred to as the Galerkin test function. The coefficients $B_{N}\left(\omega, \mathbf{q}_{\|}\right)$of the Galerkin expansion are determined by projecting the stochastic differential equation [Eq. (47)] onto the elements of the polynomial basis, $z^{N}\left(z^{2}-1\right)$, used to express $\delta \omega_{z}$ itself. This procedure requires solving the system of algebraic equations,

$$
\sum_{M} X_{N M}\left(\omega, \mathbf{q}_{\|}\right) B_{M}\left(\omega, \mathbf{q}_{\|}\right)=F_{2, N}\left(\omega, \mathbf{q}_{\|}\right),
$$

where

$$
\begin{gathered}
X_{N M}=\int_{-1}^{1} d z\left(z^{2}-1\right) z^{N}\left\{\mathcal{S} \cdot\left(z^{2}-1\right) z^{M}\right\} \\
F_{2, N}=\int_{-1}^{1} d z\left(z^{2}-1\right) z^{N} F_{2}\left(\omega, \mathbf{q}_{\|}, z\right) .
\end{gathered}
$$

To shorten notation, we are no longer denoting the $\left(\omega, \mathbf{q}_{\|}\right)$ dependence of $X_{N M}, F_{2, N}$, and the Squire operator $\mathcal{S}$ explicitly.

The Galerkin approximation is only practical if series (50) can be truncated after a few terms, so that set (51) of linear algebraic equations has a low enough dimensionality to be readily inverted and solved for $B_{N}\left(\omega, \mathbf{q}_{\|}\right)$. If we truncate Galerkin expansion (50) retaining only the first two terms, we obtain for the matrix appearing on the left-hand side (LHS) of Eq. (51),

$$
X\left(\omega, \mathbf{q}_{\|}\right)=\frac{16}{105}\left[\begin{array}{cc}
7\left[i \omega+\frac{1}{\left.\operatorname{Re}\left(q_{\|}^{2}+\frac{5}{2}\right)\right]}\right. & i q_{x} \\
i q_{x} & i \omega+\frac{1}{\operatorname{Re}}\left(q_{\|}^{2}+\frac{21}{2}\right)
\end{array}\right],
$$

which, indeed, can be readily inverted.

From Eq. (50) we see that the autocorrelation function $\left\langle\delta \omega_{z}^{*}\left(\omega, \mathbf{q}_{\|}, z\right) \cdot \delta \omega_{z}\left(\omega^{\prime}, \mathbf{q}_{\|}, z^{\prime}\right)\right\rangle$ will depend on the correlation functions $\left\langle B_{N}^{*}\left(\omega, \mathbf{q}_{\|}\right) \cdot B_{M}\left(\omega^{\prime}, \mathbf{q}_{\|}^{\prime}\right)\right\rangle$ among the coefficients to be obtained from solving Eq. (51). These correlation functions, in turn, will depend on the random-force autocorrelation function $\left\langle F_{2}^{*}\left(\omega, \mathbf{q}_{\|}, z\right) \cdot F_{2}\left(\omega^{\prime}, \mathbf{q}_{\|}^{\prime}, z^{\prime}\right)\right\rangle$. From Eq. (49) we see that the random-force autocorrelation function contains contributions from the Fourier-transformed wall-normalvelocity fluctuations $\delta v_{z}\left(\omega, \mathbf{q}_{\|}, z\right)$ and from the Fouriertransformed thermal noise $R_{z}\left(\omega, \mathbf{q}_{\|}, z\right)$. The cross correlation between $\delta v_{z}$ and $R_{z}$ is zero. Indeed, $\delta v_{z}\left(\omega, \mathbf{q}_{\|}, z\right)$ is proportional to $F_{z}\left(\omega, \mathbf{q}_{\|}, z\right)$ [36], which is uncorrelated with $R_{z}\left(\omega, \mathbf{q}_{\|}, z\right)$ in accordance with Eq. (18) (that remains valid when the Fourier transforms are applied only in the horizontal $x-y$ plane).

Using a Galerkin approximation with appropriate test functions, we have found that the solution of the stochastic
Orr-Sommerfeld equation for the autocorrelation function of the wall-normal-velocity fluctuations has the form [36]

$$
\begin{aligned}
& \left\langle\delta v_{z}^{*}\left(\omega, \mathbf{q}_{\|}, z\right) \cdot \delta v_{z}\left(\omega^{\prime}, \mathbf{q}_{\|}^{\prime}, z^{\prime}\right)\right\rangle \\
& \quad=C_{z z}\left(\omega, \mathbf{q}_{\|}, z, z^{\prime}\right)(2 \pi)^{3} \delta\left(\omega-\omega^{\prime}\right) \delta\left(\mathbf{q}_{\|}-\mathbf{q}_{\|}^{\prime}\right)
\end{aligned}
$$

with an explicit second-order Galerkin approximation for the function $C_{z z}\left(\omega, \mathbf{q}_{\|}, z, z^{\prime}\right)$ that is not repeated here.

Similarly, combining Eq. (54) with the definition [Eq. (52b)] of the projections of the random force acting in the RHS of the Squire equation, it can be shown that

$$
\begin{aligned}
& \left\langle F_{2, N}^{*}\left(\omega, \mathbf{q}_{\|}\right) \cdot F_{2, M}\left(\omega^{\prime}, \mathbf{q}_{\|}^{\prime}\right)\right\rangle \\
& \quad=\left[C_{N M}^{(1)}\left(\mathbf{q}_{\|}\right)+C_{N M}^{(2)}\left(\omega, \mathbf{q}_{\|}\right)\right](2 \pi)^{3} \delta\left(\omega-\omega^{\prime}\right) \delta\left(\mathbf{q}_{\|}-\mathbf{q}_{\|}^{\prime}\right),
\end{aligned}
$$

where the random-force correlation matrix has been split in two parts. The first part $C_{N M}^{(1)}\left(\mathbf{q}_{\|}\right)$comes from the autocorrelation of the random force $R_{z}\left(\omega, \mathbf{q}_{\|}, z\right)$ in Eq. (49) and does not depend explicitly on the frequency. This first contribution can be evaluated by following the procedures developed in previous publications [19,36] for the Rayleigh-Bénard problem and for the normal-wall velocity fluctuations in planar Couette flow, respectively. A second-order calculation gives

$$
\mathbf{C}^{(1)}\left(\mathbf{q}_{\|}\right)=\tilde{S} \frac{32 q_{\|}^{2}}{105}\left[\begin{array}{cc}
7\left(q_{\|}^{2}+\frac{5}{2}\right) & 0 \\
0 & q_{\|}^{2}+\frac{21}{2}
\end{array}\right] \text {. }
$$

The second part $\mathbf{C}^{(2)}\left(\omega, \mathbf{q}_{\|}\right)$of the correlation matrix in Eq. (55) comes from the vertical-velocity autocorrelation in Eq. (49). It does depend on the frequency and is related to the vertical integration of the function $C_{z z}\left(\omega, \mathbf{q}_{\|}, z, z^{\prime}\right)$ in Eq. (54) such that

$$
\begin{aligned}
C_{N M}^{(2)}\left(\omega, \mathbf{q}_{\|}\right)= & q_{y}^{2} \int_{-1}^{1} d z_{1} \int_{-1}^{1} d z_{2}\left(z_{1}^{2}-1\right) \\
& \times\left(z_{2}^{2}-1\right) z_{1}^{N} z_{2}^{M} C_{z z}\left(\omega, \mathbf{q}_{\|}, z_{1}, z_{2}\right) .
\end{aligned}
$$

Substitution of the second-order Galerkin approximation for $C_{z z}\left(\omega, \mathbf{q}_{\|}, z_{1}, z_{2}\right)$ into Eq. (57) yields an explicit expression for $\mathbf{C}^{(2)}\left(\omega, \mathbf{q}_{\|}\right)$. However, since this expression is very long, it is not presented here although it will be used in the sequel. It is obvious that decomposition (55) of the correlation matrix into two parts corresponds to the two different modecoupling mechanisms in the Squire equation as explained in Sec. IV.

Now, we have all the information required to evaluate the autocorrelation function of the normal-wall vorticity fluctuations including confinement effects. Similarly to Eq. (54) for wall-normal fluctuations, we find here that

$$
\begin{aligned}
& \left\langle\delta \omega_{z}^{*}\left(\omega, \mathbf{q}_{\|}, z\right) \delta \omega_{z}\left(\omega^{\prime}, \mathbf{q}_{\|}^{\prime}, z^{\prime}\right)\right\rangle \\
& \quad=W_{z z}\left(\omega, \mathbf{q}_{\|}, z, z^{\prime}\right)(2 \pi)^{3} \delta\left(\omega-\omega^{\prime}\right) \delta\left(\mathbf{q}_{\|}-\mathbf{q}_{\|}^{\prime}\right),
\end{aligned}
$$

where the function $W_{z z}\left(\omega, \mathbf{q}_{\|}, z_{1}, z_{2}\right)$ again is a sum of two contributions corresponding to the two mode-coupling mechanisms, 


$$
\begin{gathered}
W_{z z}\left(z_{1}, z_{2}\right)=W_{z z}^{(1)}\left(z_{1}, z_{2}\right)+W_{z z}^{(2)}\left(z_{1}, z_{2}\right), \\
W_{z z}^{(1)}\left(z_{1}, z_{2}\right)=\left(z_{1}^{2}-1\right)\left(z_{2}^{2}-1\right) C_{I J}^{(1)} z_{1}^{N} z_{2}^{M} X_{N I}^{-1 *} X_{M J}^{-1}, \\
W_{z z}^{(2)}\left(z_{1}, z_{2}\right)=\left(z_{1}^{2}-1\right)\left(z_{2}^{2}-1\right) C_{I J}^{(2)} z_{1}^{N} z_{2}^{M} X_{N I}^{-1 *} X_{M J}^{-1},
\end{gathered}
$$

where summation over repeated indices is understood and where the $\left(\omega, \mathbf{q}_{\|}\right)$dependence has not been indicated explicitly.

Inverting the matrix in Eq. (53) and using expressions (56) and (57) one can in principle determine the frequencydependent autocorrelation function for the wall-normalvorticity fluctuations. However, as mentioned in Sec. IV A, to determine the nonequilibrium energy amplification we only need the equal-time-correlation function averaged over the height of the fluid layer. This equal-time-correlation function can be obtained by applying a double inverse Fourier transform in the frequencies $\omega$ and $\omega^{\prime}$ to Eq. (59). If we then integrate over the vertical components $z$ and $z^{\prime}$ and introduce a factor $\frac{1}{2}$ to maintain consistency with the halfheight of the layer as reducing parameter, we obtain an expression of the form $W_{z z}\left(\mathbf{q}_{\|}\right)(2 \pi)^{2} \delta\left(\mathbf{q}_{\|}-\mathbf{q}_{\|}^{\prime}\right)$ with

$$
W_{z z}\left(\mathbf{q}_{\|}\right)=\frac{1}{4 \pi} \int_{-\infty}^{\infty} d \omega \int_{-1}^{1} d z_{1} \int_{-1}^{1} d z_{2} W_{z z}\left(\omega, \mathbf{q}_{\|}, z_{1}, z_{2}\right) .
$$

Of course, as the dynamic $W_{z z}\left(\omega, \mathbf{q}_{\|}, z_{1}, z_{2}\right)$ [Eq. (59)] splits into two parts, the static $W_{z z}\left(\mathbf{q}_{\|}\right)$splits into two parts accordingly. Let us consider each part separately. The first contribution can be evaluated from the explicit expressions of the inverse linear-response function [Eq. (53)] and the correlation matrix [Eq. (56)]. By substitution of this information into the second line of Eq. (59) and by performing the integrals as required by Eq. (60), we obtain

$$
W_{z z}^{(2)}\left(\mathbf{q}_{\|}\right)=\tilde{S} \operatorname{Re} \frac{10}{12} q_{\|}^{2} \equiv W_{z z}^{(\mathrm{E})}\left(\mathbf{q}_{\|}\right) .
$$

Therefore, just as in Eq. (43a) for the "bulk" fluctuations, we find again that the self-coupling of the vorticity fluctuations does not yield any nonequilibrium contribution to the intensity of the wall-normal-vorticity fluctuations. The difference of about $17 \%$ between Eqs. (43a) and (61) is a consequence of us having retained only two terms in the Galerkin expansion. Actually, it can be shown that the self-coupling will never yield any nonequilibrium contribution to the intensity of the wall-normal-vorticity fluctuations, independent of any Galerkin approximation. However, this physical result is not true for the dynamic structure factor $W_{z z}^{(2)}\left(\omega, \mathbf{q}_{\|}, z, z^{\prime}\right)$, for which the self-coupling mechanism in the Squire equation does induce a nonequilibrium contribution.

We conclude that the nonequilibrium contribution to the wall-normal-vorticity fluctuations solely arises from the second term in Eq. (55), i.e., from the coupling between the vorticity fluctuations and the velocity fluctuations. From the information presented it is possible to obtain the solution for a second-order Galerkin approximation to $W_{z z}^{\mathrm{NE}}\left(\mathbf{q}_{\|}\right)$. In Fig. 2 we show the solution for $q_{z}=0, q_{x}=q_{y}(\phi=\pi / 4)$ and for two values of the Reynolds number, namely, $\mathrm{Re}=50$ and $\mathrm{Re}$ $=800$. The overall shape of $W_{z z}^{\mathrm{NE}}\left(\mathbf{q}_{\|}\right)$does not depend

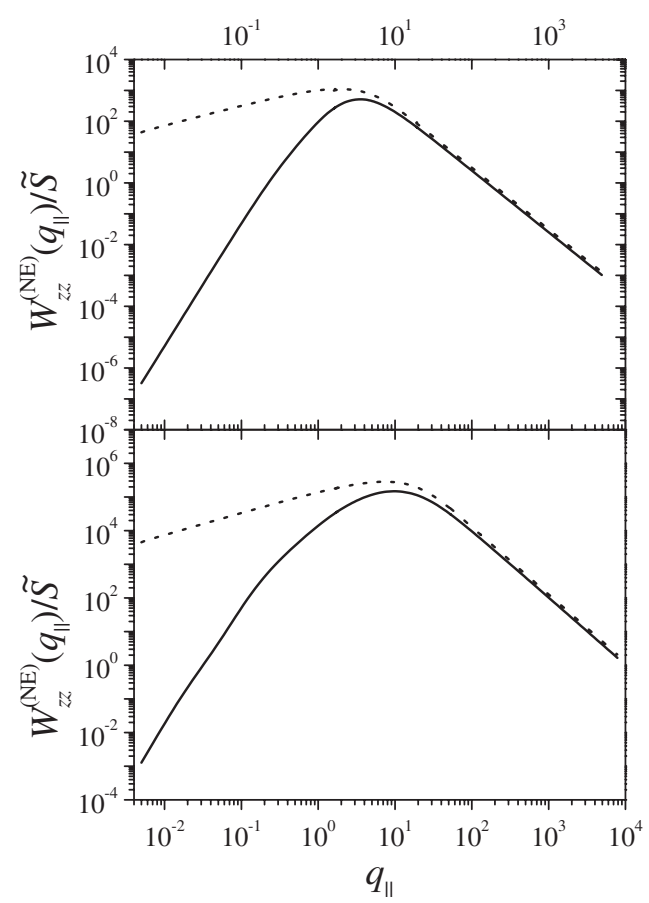

FIG. 2. Normalized intensity $W_{z z}^{\mathrm{NE}}\left(\mathbf{q}_{\|}\right) / \tilde{S}$ of the nonequilibrium wall-normal-vorticity fluctuations as a function of the horizontal wave number for $q_{z}=0, q_{x}=q_{y}(\phi=\pi / 4)$. The dotted curve represents the bulk solution given by Eq. (43b). The solid curve represents the second-order Galerkin approximation incorporating the boundary conditions. Upper panel is for $\mathrm{Re}=50$ and lower panel for $\mathrm{Re}=800$.

strongly on the Reynolds number. However, as can be seen from the lower panel in Fig. 2, when the factor $\operatorname{Re} \cos \phi$ is sufficiently large, we observe a shoulder at wave numbers $q_{\|}$ below where $W_{z z}^{\mathrm{NE}}\left(\mathbf{q}_{\|}\right)$passes through a maximum. This shoulder may be a reminiscence of the algebraic wavenumber dependence of the bulk solution (44a). However, a final resolution of this feature would require a more exact evaluation of the intensity of the nonequilibrium vorticity fluctuations.

It is possible to obtain simple analytic expressions for the intensity of the nonequilibrium wall-normal-vorticity fluctuations for small and large wave numbers,

$$
\begin{aligned}
& W_{z z}^{(\mathrm{NE})}\left(\mathbf{q}_{\|}\right) \stackrel{q_{\|} \rightarrow 0}{\rightarrow} \widetilde{S} \frac{3 \mathrm{Re}^{3}}{364} q_{\|}^{4} \sin ^{2} \phi+O\left(q_{\|}^{6}\right), \\
& W_{z z}^{(\mathrm{NE})}\left(\mathbf{q}_{\|}\right) \stackrel{q_{\|} \rightarrow \infty}{\rightarrow} \widetilde{S} \frac{45 \mathrm{Re}^{3}}{112 q_{\|}^{2}} \sin ^{2} \phi+O\left(q_{\|}^{-4}\right),
\end{aligned}
$$

to be compared with Eq. (44) for the wave-number dependence of the wall-normal-vorticity fluctuations in the absence of boundary conditions. As is to be expected $[1,36]$, a comparison between Eqs. (44a) and (62a) confirms that the boundary conditions profoundly affect the intensity of the nonequilibrium vorticity fluctuations at small wave numbers. The asymptotic behavior of the intensity at large wave numbers is unaffected by the boundary conditions. The small difference of the coefficient $45 / 112 \simeq 0.402$ in Eq. (62b) 
from the exact value of 0.5 in Eq. (44b) shows that our two-term Galerkin expansion yields a good approximation for the intensity of the nonequilibrium wall-normal-vorticity fluctuations.

From Fig. 2 we see that the intensity $W_{z z}^{(\mathrm{NE})}\left(\mathbf{q}_{\|}\right)$exhibits a simple crossover between the two asymptotic limits, represented by Eq. (44), except for a shoulder at large values of $\operatorname{Re} \cos \phi$. While the complete mathematical solution for $W_{z z}^{(\mathrm{NE})}\left(\mathbf{q}_{\|}\right)$is very complex, we have found that the solution can be represented by a simple approximant of the form

$$
W_{z z}^{(\mathrm{NE})}\left(\mathbf{q}_{\|}\right) \simeq \frac{\tilde{S} \operatorname{Re}^{3} \frac{3}{364} q_{\|}^{2} \sin ^{2} \phi}{1+a(\operatorname{Re} \cos \phi)^{2} q_{\|}^{2}+b(\operatorname{Re} \cos \phi)^{2} q_{\|}^{4}+\frac{4}{195} q_{\|}^{6}}
$$

with $a=1.9 \times 10^{-4}$ and $b=1.8 \times 10^{-5}$. This approximant for $W_{z z}^{(\mathrm{NE})}\left(\mathbf{q}_{\|}\right)$reproduces the limiting behavior of the solution as given by Eq. (62a), while the parameters $a$ and $b$ have been chosen so that the approximant also accounts for the presence of a shoulder of the type seen in the lower panel of Fig. 2. We have numerically checked that approximant (63) never deviates from the numerical solution by more than $10 \%$. Hence, instead of the mathematical solution for the Galerkin approximation we shall use in the sequel approximant (63) to represent $W_{z z}^{(\mathrm{NE})}\left(\mathbf{q}_{\|}\right)$.

\section{NONEQUILIBRIUM ENERGY AMPLIFICATION}

A very interesting property of laminar flows is the kinetic energy associated with the intrinsic thermally excited velocity fluctuations. We shall first evaluate the kinetic-energy enhancement due to the nonequilibrium velocity fluctuations in the bulk and then consider the effect of the boundary conditions on the kinetic-energy enhancement.

\section{A. Energy amplification in the absence of boundary conditions}

To determine the nonequilibrium enhancement of the kinetic energy, we need the autocorrelation functions of all the fluctuating-velocity components. Inverting the relationship $\delta \omega_{z}(\mathbf{q}, t)=i q_{x} \delta v_{y}(\mathbf{q}, t)-i q_{y} \delta v_{x}(\mathbf{q}, t)$ and using the divergence-free condition for the velocity fluctuations, we can relate the kinetic energy to the intensities of the wallnormal-velocity and vorticity fluctuations,

$$
\begin{aligned}
\left\langle\delta v_{i}^{*}(\mathbf{q}, t) \cdot \delta v_{i}\left(\mathbf{q}^{\prime}, t\right)\right\rangle= & \frac{q^{2}}{q_{\|}^{2}}\left\langle\delta v_{z}^{*}(\mathbf{q}, t) \delta v_{z}\left(\mathbf{q}^{\prime}, t\right)\right\rangle \\
& +\frac{1}{q_{\|}^{2}}\left\langle\delta \omega_{z}^{*}(\mathbf{q}, t) \delta \omega_{z}\left(\mathbf{q}^{\prime}, t\right)\right\rangle,
\end{aligned}
$$

where summation over the index $i$ is understood. Upon substituting Eqs. (35) and (36) into the RHS of Eq. (64) and using Eqs. (37)-(42), we see that the kinetic energy associated with the fluctuations can be written as

$$
\left\langle\delta v_{i}^{*}(\mathbf{q}, t) \cdot \delta v_{i}\left(\mathbf{q}^{\prime}, t\right)\right\rangle=K(\mathbf{q})(2 \pi)^{3} \delta\left(\mathbf{q}-\mathbf{q}^{\prime}\right) .
$$

The intensity $K(\mathbf{q})$ of the kinetic-energy fluctuations is the sum of an equilibrium contribution $2 \widetilde{S} R$ e and a nonequilibrium contribution $K_{\mathrm{NE}}(\mathbf{q})$,

$$
K(\mathbf{q})=2 \tilde{S} \operatorname{Re}+K_{\mathrm{NE}}(\mathbf{q}),
$$

where

$$
\tilde{K}_{\mathrm{NE}}(\mathbf{q})=\frac{q^{2}}{q_{\|}^{2}} C_{z z}^{(\mathrm{NE})}(\mathbf{q})+\frac{1}{q_{\|}^{2}} W_{z z}^{(\mathrm{NE})}(\mathbf{q}),
$$

with $C_{z z}^{(\mathrm{NE})}(\mathbf{q})$ and $W_{z z}^{(\mathrm{NE})}(\mathbf{q})$ given by Eqs. (39) and (43b), respectively.

The energy amplification of the system under shear can be readily obtained by applying a double inverse Fourier transformation (in $\mathbf{q}$ and $\mathbf{q}^{\prime}$ ) to Eq. (65) and substituting the result into the dimensionless version of Eq. (11),

$$
\left\langle[\overline{\delta \mathbf{v}}(t)]^{2}\right\rangle=\frac{1}{\tilde{V}^{2}} \iint_{\tilde{V} \times \tilde{V}} d \mathbf{r} d \mathbf{r}^{\prime} \int_{\mathrm{R}^{3}} \frac{d \mathbf{q}}{(2 \pi)^{3}} K(\mathbf{q}) e^{i \mathbf{q}\left(\mathbf{r}-\mathbf{r}^{\prime}\right)},
$$

where the dimensionless averaging volume $\tilde{V}$ equals the physical volume $V$ divided by $L^{3}$. If we substitute the equilibrium part of $K(\mathbf{q})$ into Eq. (68) we reproduce the dimensionless version of the equilibrium result, given by Eq. (12), namely, $\left\langle[\overline{\delta \mathbf{v}}(t)]^{2}\right\rangle_{\mathrm{E}}=2 \tilde{S} \operatorname{Re} / \tilde{V}$. The nonequilibrium term $\widetilde{K}_{\mathrm{NE}}(\mathbf{q})$ in Eq. (66) implies the existence of a nonequilibrium enhancement of the kinetic energy in a system under shear, a phenomenon commonly referred to as energy amplification in the fluid-dynamics literature. Substituting Eq. (67) into Eq. (68), we obtain for the nonequilibrium energy amplification

$$
\left\langle[\overline{\delta \mathbf{v}}(t)]^{2}\right\rangle_{\mathrm{NE}}=\frac{1}{(2 \pi)^{3}} \int_{\mathbb{R}^{3}} d \mathbf{q} K_{\mathrm{NE}}(\mathbf{q}),
$$

where the limit $\widetilde{V} \rightarrow 0$ has been taken.

The integral in Eq. (69) can be readily evaluated without any need of introducing a cutoff for small values of the wave number. Specifically, the anisotropic nature of the nonequilibrium fluctuations in a system under shear causes the divergent $1 / q^{2}$ terms in the asymptotic expansion for large $q$ of $C_{z z}^{(\mathrm{NE})}(\mathbf{q})$ to cancel on integration over the azimuthal angle $\phi$. Therefore, the first terms with a nonzero contribution in the large- $q$ limit are proportional to $q^{-4}$ and give meaningful results when integrated over the wave number $\mathbf{q}$, as dictated by Eq. (69). This issue has been discussed in detail in our previous publication on the subject [36] and also by Wada and Sasa [38].

Substituting Eq. (67) into Eq. (69) and performing the integration over $\mathrm{R}^{3}$, we obtain

$$
\left\langle[\overline{\delta \mathbf{v}}(t)]^{2}\right\rangle_{\mathrm{NE}}=\tilde{S} \operatorname{Re}\left\{I_{\mathrm{OS}}+I_{\mathrm{S}}\right\}(\operatorname{Re})^{3 / 2},
$$

where the coefficients $I_{\mathrm{OS}} \simeq 0.0126$ and $I_{\mathrm{S}} \simeq 0.100$ result from the integration over $\mathbb{R}^{3}$ of $C_{z z}(\mathbf{q})$ (obtained from the solution of the Orr-Sommerfeld equation) and $W_{z z}(\mathbf{q})$ (ob- 
tained from the solution of the Squire equation), respectively. It is interesting to note that the contribution from the Squire equation is much more important than that from the OrrSommerfeld equation, a feature that will remain true when the effect of the boundary conditions will be incorporated.

To interpret Eq. (70) it is convenient to revert to dimensional physical variables. By using Eq. (27) for $\widetilde{S}$, Eq. (16) for Re, Eq. (13) for the velocities, and adding the equilibrium contribution we find

$$
\left\langle[\overline{\delta \mathbf{v}}(t)]^{2}\right\rangle=\frac{k_{B} T}{\rho_{0}}\left[\frac{2}{V}+0.1126\left(\frac{|\dot{\gamma}|}{\nu}\right)^{3 / 2}\right],
$$

which does not depend explicitly on $L$, as is to be expected when boundary conditions are not considered. It is interesting to note from Eq. (71) that the equilibrium contribution does depend on the averaging volume $V$ as in Eq. (12), while the nonequilibrium contribution does not. This feature is a consequence of the spatially long-ranged nature of the nonequilibrium fluctuations. We also observe that, within the linear theory presented in this paper, the nonequilibrium energy amplification increases with the shear rate to the power $3 / 2$. Of course, the energy amplification cannot increase unboundedly with the shear rate and should eventually become saturated for large shear rates by some nonlinear mechanism, probably related to the onset of turbulence.

\section{B. Energy amplification in the presence of boundary conditions}

To accommodate the boundary conditions we apply, just as in Sec. V, a spatial Fourier transformation in the plane parallel to the boundaries. Then, instead of Eq. (64), the relationship between the equal-time-correlation functions becomes

$$
\begin{aligned}
\left\langle\delta v_{i}^{*}\left(\mathbf{q}_{\|}, z\right) \delta v_{i}\left(\mathbf{q}_{\|}^{\prime}, z^{\prime}\right)\right\rangle= & \left\langle\delta v_{z}^{*}\left(\mathbf{q}_{\|}, z\right) \delta v_{z}\left(\mathbf{q}_{\|}^{\prime}, z^{\prime}\right)\right\rangle \\
& +\frac{1}{q_{\|}^{2}}\left\{\partial_{z} \partial_{z^{\prime}}\left\langle\delta v_{z}^{*}\left(\mathbf{q}_{\|}, z\right) \delta v_{z}\left(\mathbf{q}_{\|}^{\prime}, z^{\prime}\right)\right\rangle\right. \\
& \left.+\left\langle\delta \omega_{z}^{*}\left(\mathbf{q}_{\|}, z\right) \delta \omega_{z}\left(\mathbf{q}_{\|}^{\prime}, z^{\prime}\right)\right\rangle\right\}
\end{aligned}
$$

at any time $t$. Note that, just as in Eqs. (54) and (58), all correlation functions in Eq. (72) are proportional to $\delta\left(\mathbf{q}_{\|}-\mathbf{q}_{\|}^{\prime}\right)$.

A major effect of the boundary conditions is that they break the translational symmetry of the correlation functions along the wall-normal $z$ axis. Thus the equal-time-correlation functions in Eq. (72) not only depend on the distance $\left|z-z^{\prime}\right|$ but also on the average position $\left(z+z^{\prime}\right) / 2$. Hence, in evaluating the energy amplification we need to specify the spatial position $z$ where the volume element $V$ for taking the average is located. One simple possibility would be to evaluate the energy amplification at midheight, i.e., for $z=z^{\prime}=0$. However, physically more relevant is the energy amplification averaged over the height of the fluid layer. We thus extend the volume $V$ for averaging the velocity fluctuations in Eq. (10) to the full height of the fluid layer (i.e., in physical units from $z=-L$ to $z=+L$ ) but with a small surface area $A$ in the horizontal $x-y$ plane. This shape of averaging volume $V$ corresponds to the volumes probed in lightscattering or shadowgraph experiments $[1,19]$. Due to the boundary conditions, with this choice of volume element the middle term in the RHS of Eq. (72), containing derivatives of the wall-normal-velocity-correlation function, does not contribute to the average energy amplification.

To determine the (average) energy amplification we apply a two-dimensional inverse Fourier transformation in $\mathbf{q}_{\|}$and $\mathbf{q}_{\|}^{\prime}$ and then average over the volume element specified above. Using the information collected in Sec. $\mathrm{V}$ for the velocity and vorticity correlation functions, we conclude that this energy amplification can be expressed as

$$
\left\langle[\overline{\delta \mathbf{v}}(t)]^{2}\right\rangle=\frac{1}{2 \widetilde{A}^{2}} \iint_{\tilde{A} \times \tilde{A}} d \mathbf{r}_{\|} d \mathbf{r}_{\|}^{\prime} \int_{\mathbb{R}^{2}} \frac{d \mathbf{q}_{\|}}{(2 \pi)^{2}} K\left(\mathbf{q}_{\|}\right) e^{i \mathbf{q}_{\|}\left(\mathbf{r}_{\|}-\mathbf{r}_{\|}^{\prime}\right)},
$$

where

$$
K\left(\mathbf{q}_{\|}\right)=C_{z z}\left(\mathbf{q}_{\|}\right)+\frac{1}{q_{\|}^{2}} W_{z z}\left(\mathbf{q}_{\|}\right)
$$

with $W_{z z}\left(\mathbf{q}_{\|}\right)$defined by Eq. (60) and $C_{z z}\left(\mathbf{q}_{\|}\right)$by Eq. (39) in our previous publication on the subject [36]. The prefactor in the LHS of Eq. (73) includes the inverse dimensionless horizontal area $\tilde{A}$ of the averaging volume times the height ( 2 in dimensionless units). The height does not appear squared because a factor 2 was already included in the definition of $W_{z z}\left(\mathbf{q}_{\|}\right)$and $C_{z z}\left(\mathbf{q}_{\|}\right)$. This definition facilitates a comparison between results obtained with and without boundary conditions, as will be explained in Sec. VI C.

As a next step we need to perform the integration in Eq. (73). For this purpose it is convenient to decompose $K\left(\mathbf{q}_{\|}\right)$, defined by Eq. (74), into an equilibrium and a nonequilibrium contribution. Combining Eq. (61) with Eq. (41a) in Ref. [36] we find for the equilibrium contribution,

$$
K_{\mathrm{E}}\left(\mathbf{q}_{\|}\right)=\tilde{S} \operatorname{Re}\left\{\frac{7}{10} \frac{q_{\|}^{2}}{q_{\|}^{2}+3}+\frac{10}{12}\right\} .
$$

Upon substituting Eq. (75) into Eq. (73), performing the integrations, and reverting the result to physical dimensions, we obtain

$$
\left\langle[\overline{\delta \mathbf{v}}(t)]^{2}\right\rangle_{\mathrm{E}} \simeq \frac{23}{15} \frac{k_{B} T}{\rho 2 L A},
$$

where we display only the leading term in the limit of averaging over a small horizontal surface $A$. In that case we obtain from our second-order approximation a result that is approximately $20 \%$ off from the exact expected result $(23 / 15 \simeq 1.533$ to be compared with 2$)$.

The nonequilibrium energy amplification can be evaluated by a similar procedure, namely, by substituting into Eq. (74) the nonequilibrium part of $C_{z z}\left(\mathbf{q}_{\|}\right)$, given by Eq. (41b) in Ref. [36], and the nonequilibrium part of $W_{z z}\left(\mathbf{q}_{\|}\right)$, given by Eq. (63). In this case, the integration over $\mathbf{q}_{\|}$in Eq. (73) is always convergent (no short-ranged fluctuations) and the limit can be taken even before integrating over the wave vector. Hence, instead of Eq. (73), one can directly use the more convenient expression 


$$
\left\langle[\overline{\delta \mathbf{v}}(t)]^{2}\right\rangle_{\mathrm{NE}}=\frac{1}{2} \int_{\mathbb{R}^{2}} \frac{d \mathbf{q}_{\|}}{(2 \pi)^{2}}\left\{C_{z z}^{(\mathrm{NE})}\left(\mathbf{q}_{\|}\right)+\frac{W_{z z}^{(\mathrm{NE})}\left(\mathbf{q}_{\|}\right)}{q_{\|}^{2}}\right\} .
$$

Integral (77) cannot be performed analytically for arbitrary Reynolds numbers. However, the asymptotic limit for large Re can be evaluated explicitly and we obtain in terms of the dimensional physical variables,

$$
\left\langle[\overline{\delta \mathbf{v}}(t)]^{2}\right\rangle_{\mathrm{NE}}^{\mathrm{Re} \rightarrow \infty} \rightarrow \frac{k_{B} T}{\rho_{0}}\left[\frac{1.43 \ln \operatorname{Re}-4.28}{L^{3}}+0.47 \frac{|\dot{\gamma}|}{\nu L}\right],
$$

where the first two terms arise from the solution of the stochastic Orr-Sommerfeld equation and the third term from the stochastic Squire equation. Hence, just as in the absence of boundary conditions, we find that the contribution from the Squire equation is much more important than the contribution from the Orr-Sommerfeld equation.

\section{Comparison of energy amplification with and without confinement}

An accurate quantitative analysis of the effect of confinement on the energy amplification cannot be obtained from a direct comparison between Eqs. (71) and (78) for the energy amplification with and without boundary conditions because of the different averaging volume used in the two cases. For such a comparison we re-evaluate integral (68) for the bulk energy amplification over the same volume $V$ used in the evaluation of integral (73) for the energy amplification in the presence of boundary conditions. For this purpose we approximate the vertical integral in Eq. (68) as

$$
\frac{1}{4(2 \pi)} \int_{-1}^{1} d z_{1} \int_{-1}^{1} d z_{2} e^{i q_{z}\left(z_{1}-z_{2}\right)}=\frac{1}{2} \delta\left(q_{z}\right)
$$

where we have assumed that the integration interval ([-L,L] in physical units) for $z_{1}$ can be extended to infinity, so that a delta function of $q_{z}$ is obtained. If we then substitute Eq. (79) into Eq. (68) we obtain for the bulk energy amplification averaged over a volume element that comprises the full height of the layer,

$$
\begin{aligned}
\left\langle[\overline{\delta \mathbf{v}}(t)]^{2}\right\rangle^{(\mathrm{B})}= & \frac{1}{2 \widetilde{A}^{2}} \iint_{\tilde{A} \times \tilde{A}} d \mathbf{r}_{\|} d \mathbf{r}_{\|}^{\prime} \int_{\mathbb{R}^{2}} \frac{d \mathbf{q}_{\|}}{(2 \pi)^{2}} K^{(\mathrm{B})} \\
& \times\left(\mathbf{q}_{\|}, q_{z}=0\right) e^{i \mathbf{q}_{\|}\left(\mathbf{r}_{\|}-\mathbf{r}_{\|}^{\prime}\right)} .
\end{aligned}
$$

Here we have introduced a superscript B for bulk to indicate that Eq. (66) associated with the bulk fluctuations needs to be substituted into Eq. (80). Comparing Eq. (80) with Eq. (73), we observe that we are now evaluating the energy amplification without confinement by an averaging procedure completely parallel to the evaluation of the energy amplification with confinement in Sec. VI B. We note that the factor $1 / 2$ appearing before the delta function in Eq. (79) is the reason why a similar factor $1 / 2$ arising from the dimensionless height of the layer was included in definition (60) of $W_{z z}\left(\mathbf{q}_{\|}\right)$ when boundary conditions are considered. Otherwise we would have a prefactor $1 / 4 \widetilde{A}^{2}$ in Eq. (73) in contrast to the prefactor $1 / 2 \widetilde{A}^{2}$ in Eq. (80). Equation (80) also explains why the energy amplification in a confined layer has to be compared with the intensity of the bulk energy amplification at $q_{z}=0$, as was done in Fig. 2 for the wall-normal-vorticity fluctuations and in our previous publication [36] for the wallnormal-velocity fluctuations.

For the nonequilibrium part of integral (80) the limit $\widetilde{A} \rightarrow 0$ can be taken, just as in Eq. (77). Then Eq. (80) reduces to a two-dimensional integral that has two contributions, one from $C_{z z}^{(\mathrm{NE})}\left(\mathbf{q}_{\|}, 0\right)$, given by Eq. (39), and the other from $W_{z z}^{(\mathrm{NE})}\left(\mathbf{q}_{\|}, 0\right)$, given by Eq. (43). The first contribution to the integral can be evaluated easily. The evaluation of the second contribution is more involved since the integrand diverges at $q_{z}=0$, as is evident from Eq. (45). This complication can be solved by evaluating the leading term when $q_{z} \rightarrow 0$ of Eq. (80), which is $\propto q_{z}^{-1 / 2}$, and then substitute it into the LHS of Eq. (79). We skip any further details but quote the final results in terms of dimensional physical variables,

$$
\left\langle[\overline{\delta \mathbf{v}}(t)]^{2}\right\rangle_{\mathrm{NE}}^{(\mathrm{B})}=\frac{k_{B} T}{\rho_{0}}\left\{\frac{\sqrt{3}}{4 \pi} \frac{\dot{\gamma}}{\nu L}+\frac{0.095}{\sqrt{L}}\left(\frac{|\dot{\gamma}|}{\nu}\right)^{5 / 4}\right\} .
$$

The first term in Eq. (81) arises from the solution of the Orr-Sommerfeld equation and the second one from the solution of the Squire equation. On comparing Eq. (81) with Eq. (71), where the velocity fluctuations were averaged over a small volume element $V$ in the absence of boundary conditions, we note that averaging the fluctuations over the full height of the layer results in some lowering of the energy amplification. Specifically, the Orr-Sommerfeld equation now yields a contribution to the nonequilibrium energy amplification that increases proportionally to $\dot{\gamma}$ instead of $\dot{\gamma}^{3 / 2}$, and the Squire equation yields a contribution that is now proportional to $\dot{\gamma}^{5 / 4}$ instead of $\dot{\gamma}^{3 / 2}$.

On comparing Eq. (78) for the layer-averaged nonequlibrium energy amplification in the presence of boundary conditions with Eq. (81) in the absence of boundary conditions, we conclude that confinement causes some reduction in the nonequilibrium energy amplification. Specifically, incorporation of the boundary conditions causes the Orr-Sommerfeld contribution to increase with the logarithm of the shear rate and the Squire contribution proportional to the shear rate itself.

\section{DISCUSSION}

In this paper we have evaluated the intrinsic nonequilibrium enhancement of the velocity fluctuations in laminar Couette flow, i.e., an enhancement that is always present as a consequence of thermal excitations. We have determined the nonequilibrium fluctuations of all velocity components by combining the solution of the stochastic Orr-Sommerfeld equation for the wall-normal-velocity fluctuations, obtained in a previous publication [36], with the solution of the stochastic Squire equation for the wall-normal-vorticity fluctuations. The nonequilibrium normal-velocity fluctuations are caused by a self-coupling of the normal-velocity fluctuations through the velocity gradient. The nonequilibrium normal- 
vorticity fluctuations are affected by two coupling mechanisms, namely, a self-coupling of the normal-vorticity fluctuations and a cross coupling of the normal-vorticity fluctuations with the normal-velocity fluctuations. However, we have found that the self-coupling of the vorticity fluctuations affects the time dependence but not the intensity of the nonequilibrium normal-vorticity fluctuations. Hence, the equal-time normal-vorticity correlation functions only result from the coupling of the normal-vorticity fluctuations with the normal-velocity fluctuations. By averaging over the fluctuations of all velocity components and over all wave-vector directions, we have also determined the net energy amplifications resulting from the intrinsic velocity fluctuations. It turns out that the solution of the stochastic Orr-Sommerfeld equation yields a minor contribution and the solution of the stochastic Squire equation yields a major contribution to the energy amplification. Hence, the dominant contribution to the energy amplification arises from the cross coupling between normal-vorticity and normal-velocity fluctuations.

We have restricted the analysis here to the equal-timecorrelation functions that represent the nonequilibrium enhancement of the intensity of the fluctuations. It would also be of interest to analyze the time dependence of the nonequilibrium fluctuations, as pointed out by several investigators $[29,34,45,46]$. The method for solving the stochastic OrrSommerfeld and Squire equations, presented in this paper, can also be used to determine the time-dependent velocitycorrelation functions. However, such an analysis is more complicated since it would require also an evaluation of the effects from the self-coupling of the normal-vorticity fluctuations.

In this paper we have obtained the solutions of the stochastic Orr-Sommerfeld and Squire equations in the presence of boundary conditions in terms of a Galerkin approximation. With the Galerkin approximation we were able to elucidate the nature of the energy amplification as affected by the boundary conditions. A more rigorous treatment in terms of the Orr-Sommerfeld and Squire operators is under investigation.

While we have investigated the nonequilibrium energy amplification associated with intrinsic thermal noise, other investigators have studied energy amplification in laminar
Couette flow and Poiseulle flow resulting from externally imposed noise, also referred to as stochastic forcing $[27,32,34]$. The physical mechanisms for the nonequilibrium energy enhancement from thermal noise or from imposed noise are very similar. This can be illustrated by comparing our results with those obtained by Bamieh and Dahleh [32] for energy amplification in channel flows with stochastic forcing. The analysis of Bamieh and Dahleh [32] was restricted to fluctuations in the spanwise direction, which, translated to our language, means fluctuations with $q_{x}=0$. From an inspection of the Fourier-transformed OrrSommerfeld [Eq. (19)] and Squire [Eq. (22)] equations one readily sees that the condition $q_{x}=0$ implies that the normalvelocity fluctuations remain the same as in equilibrium, so that only the normal-vorticity fluctuations exhibit a nonequilibrium enhancement in the spanwise direction. In our analysis the intensity $W_{z z}^{(\mathrm{NE})}\left(\mathbf{q}_{\|}\right)$of the nonequilibrium enhancement of the fluctuations in the spanwise direction is approximated by Eq. (63) with $\cos \phi=1$. We confirm the observation of Bamieh and Dahleh [32] that this quantity increases with $\mathrm{Re}^{3}$. However, since the product $\widetilde{S} \mathrm{Re}$ is independent of Re, the actual nonequilibrium enhancement increases with $\mathrm{Re}^{2}$. From our Fig. 2 we also confirm the observation of previous investigators $[27,32]$ that the maximum of the nonequilibrium enhancement of the fluctuations occurs at a dimensionless wave number of about 1.5. It is important to note that we have not only obtained the intensity of the fluctuations in the spanwise direction but in all directions. If we average over all directions we find that the net energy amplification for large Reynolds numbers varies only linearly with the Reynolds number Re or the shear rate $\dot{\gamma}_{0}$ in accordance with Eq. (78).

\section{ACKNOWLEDGMENTS}

We are indebted to Frans T. M. Nieuwstadt, who called our attention to the problem addressed in this research. We are indebted to the Spanish Ministerio de Educación y Ciencia for supporting J.V.S. during a sabbatical leave at the Universidad Complutense de Madrid where this work was initiated and for continued support through Research Project No. FIS2008-03801.
[1] J. M. Ortiz de Zárate and J. V. Sengers, Hydrodynamic Fluctuations in Fluids and Fluid Mixtures (Elsevier, Amsterdam, 2006).

[2] J. R. Dorfman, T. R. Kirkpatrick, and J. V. Sengers, Annu. Rev. Phys. Chem. 45, 213 (1994).

[3] P. N. Segrè, R. W. Gammon, J. V. Sengers, and B. M. Law, Phys. Rev. A 45, 714 (1992).

[4] P. N. Segrè, R. W. Gammon, and J. V. Sengers, Phys. Rev. E 47, 1026 (1993).

[5] W. B. Li, P. N. Segrè, R. W. Gammon, and J. V. Sengers, Physica A 204, 399 (1994).

[6] A. Vailati and M. Giglio, Phys. Rev. Lett. 77, 1484 (1996); Physica A 235, 105 (1997); Phys. Rev. E 58, 4361 (1998).
[7] W. B. Li, K. J. Zhang, J. V. Sengers, R. W. Gammon, and J. M. Ortiz de Zárate, Phys. Rev. Lett. 81, 5580 (1998); J. Chem. Phys. 112, 9139 (2000).

[8] M. Wu, G. Ahlers, and D. S. Cannell, Phys. Rev. Lett. 75, 1743 (1995).

[9] A. Vailati and M. Giglio, Nature (London) 390, 262 (1997).

[10] E. Bodenschatz, W. Pesch, and G. Ahlers, Annu. Rev. Fluid Mech. 32, 709 (2000).

[11] D. Brogioli, A. Vailati, and M. Giglio, Phys. Rev. E 61, R1 (2000).

[12] J. Oh, J. M. Ortiz de Zárate, J. V. Sengers, and G. Ahlers, Phys. Rev. E 69, 021106 (2004).

[13] F. Croccolo, D. Brogioli, A. Vailati, M. Giglio, and D. S. Can- 
nell, Phys. Rev. E 76, 041112 (2007).

[14] C. J. Takacs, G. Nikolaenko, and D. S. Cannell, Phys. Rev. Lett. 100, 234502 (2008).

[15] V. M. Zaitsev and M. I. Shliomis, Zh. Eksp. Teor. Fiz. 59, 1538 (1970). [Sov. Phys. JETP 32, 866 (1971).

[16] J. B. Swift and P. C. Hohenberg, Phys. Rev. A 15, 319 (1977).

[17] M. C. Cross and P. C. Hohenberg, Rev. Mod. Phys. 65, 851 (1993).

[18] T. R. Kirkpatrick, E. G. D. Cohen, and J. R. Dorfman, Phys. Rev. A 26, 995 (1982).

[19] J. M. Ortiz de Zárate and J. V. Sengers, Physica A 300, 25 (2001); Phys. Rev. E 66, 036305 (2002).

[20] P. G. Drazin and W. H. Reid, Hydrodynamic Stability, 2nd ed. (Cambridge University Press, Cambridge, 2004).

[21] P. J. Schmid and D. S. Henningson, Stability and Transition in Shear Flows (Springer, Berlin, 2001).

[22] S. Grossmann, Rev. Mod. Phys. 72, 603 (2000).

[23] J. Lerner and E. Knobloch, J. Fluid Mech. 189, 117 (1988).

[24] B. Dubrulle and J. Zahn, J. Fluid Mech. 231, 561 (1991).

[25] K. M. Butler and B. F. Farrell, Phys. Fluids A 4, 1637 (1992).

[26] L. N. Trefethen, A. Trefethen, S. C. Reddy, and T. A. Driscoll, Science 261, 578 (1993).

[27] B. F. Farrell and P. J. Ioannou, J. Atmos. Sci. 50, 200 (1993); Phys. Fluids A 5, 2600 (1993); Phys. Rev. Lett. 72, 1188 (1994).

[28] O. Dauchot and F. Daviaud, Phys. Fluids 7, 335 (1995).

[29] F. Waleffe, Phys. Fluids 7, 3060 (1995); 9, 883 (1997).

[30] A. Schmiegel and B. Eckhardt, Phys. Rev. Lett. 79, 5250 (1997).

[31] D. Barkley and L. S. Tuckerman, Phys. Fluids 11, 1187
(1999).

[32] B. Bamieh and M. Dahleh, Phys. Fluids 13, 3258 (2001).

[33] A. Bottaro, P. Corbett, and P. Luchini, J. Fluid Mech. 476, 293 (2003).

[34] B. Eckhardt and R. Pandit, Eur. Phys. J. B 33, 373 (2003).

[35] D. Biau and A. Bottaro, Phys. Fluids 16, 3515 (2004).

[36] J. M. Ortiz de Zárate and J. V. Sengers, Phys. Rev. E 77, 026306 (2008).

[37] J. F. Lutsko and J. W. Dufty, Phys. Rev. E 66, 041206 (2002).

[38] H. Wada and S. I. Sasa, Phys. Rev. E 67, 065302(R) (2003).

[39] L. D. Landau and E. M. Lifshitz, Fluid Mechanics (Pergamon, London, 1959); Fluid Mechanics, 2nd ed. (Pergamon, London, 1987), English version.

[40] R. Schmitz and E. G. D. Cohen, J. Stat. Phys. 39, 285 (1985).

[41] S. Chandrasekhar, Hydrodynamic and Hydromagnetic Stability (Oxford University Press, Oxford, 1961); Hydrodynamic and Hydromagnetic Stability (Dover, New York, 1981).

[42] J. P. Hansen and I. R. McDonald, Theory of Simple Liquids, 2nd ed. (Academic, London, 1986).

[43] J. P. Boon and S. Yip, Molecular Hydrodynamics (McGrawHill, New York, 1980); Molecular Hydrodynamics (Dover, New York, 1991).

[44] J. Lutsko and J. W. Dufty, Phys. Rev. A 32, 3040 (1985).

[45] J. W. Dufty and J. Lutsko, in Recent Developments in Nonequilibrium Thermodynamics: Fluids and Related Topics, Lecture Notes in Physics Vol. 253, edited by J. Casas-Vázquez, D. Jou, and J. M. Rubí (Springer, Berlin, 1986), pp. 47-84.

[46] A. Onuki, Phys. Lett. A 70, 31 (1979). 